\title{
Reproducibility of the Neutral Zone Recording on the Estimated Occlusal Plane
}

\author{
Masao Morikawa, Shinki Ryo, Toshihiro Shimizu, \\ Kazuo Yasumoto and Shizuo Toyoda \\ First Department of Prosthetic Dentistry (Chief : Prof. Shizuo Toyoda) \\ Yoshio Kozono \\ Department of Materials Science (Chief : Prof. Ichiro Hayashi) \\ Kyushu Dental College, Kitakyushu, Japan \\ Hironobu Sato \\ Second Department of Prosthetic Dentistry (Chief : Prof. Hiroyuki Fujii) \\ Nagasaki University School of Dentistry, Nagasaki, Japan \\ (Received on 25th Angust, 1983)
}

\begin{abstract}
Introduction
It is the most difficult to secure the good stability and retention of the complete denture in all the prosthodontic appliances. Especially the lower denture may become more unstable than the upper, because the mandibular basal seat is too narrow and the surrounding tissues are extremely movable. In consideration of such disadvantages, various techniques have been developed and effectively employed ${ }^{1 \sim 17}$ : interalveolar ridge line theory; Pound's technique; key zone technique; and so forth. Neutral zone technique devised by Beresin and Schiesser $(1973)^{18)}$, which is analogous in principle to flange technique by Lott and Levin $(1966)^{19)}$, has also been evaluated to be one of the most successful means to improve the stability and retention of the lower complete denture as well as the comfort of the patient. It is assumed that the neutral zone is present where the outward movements or forces of the tongue are regarded as neutralized by the inward ones of the cheeks and lips. Thus, this technique is based on the fundamental principle that the stability and retention of the denture will be substantially secured when the artificial teeth are properly arranged within the neutral zone and when the peripheral borders and polished surfaces of the denture are constructed so as to adapt intimately to the musculatures in function. The authors have progressively modified the neutral zone technique since its original was introduced by Tsubone about two decades $\mathrm{ago}^{20 \sim 22)}$. According to this technique, the space for setting the artificial teeth is contoured by the plaster matrices correspondingly to the neutral zone, and hence the teeth are only to be placed mechanically in the settled area.
\end{abstract}

Although the determination of the neutral zone is the prime factor affecting all the subsequent procedures and the performance of the denture, no fundamental inves- 
tigations could be found about the preciseness or the invariabilty of the form and shape of the zone itself. In the present study, differences in site and shape of the neutral zone were examined when the records were practically established repeatedly for several times under the same condition by an experienced practitioner. Furthermore, the experiments were carried out under altered oral conditions such as raising the bite or utilizing the accustomed upper denture.

\section{Materials and Methods}

The denture base plate was carefully fabricated on the plaster cast. The impression making in the neutral zone technique required much more elaborate functional movements of the tongue, cheeks and lips than in the conventional techniques. Materials employed for the base plate was self-curing acrylic resin "Ostron" (G-C Dental Industrial Corp., Japan). The dough of the resin was pressed on the plaster cast to a plate, about $1 \mathrm{~mm}$ uniform thick. Peripheral border of the plate was trimmed away along the border of the underlying plaster cast. The plate was then put into the mouth to inspect its propriety for this technique. Some adjustments were made when necessary. The patient, at the same time, could get accustomed to the functional movements with the plate. This plate became the original base plate, from which an impression mold was prepared for duplication. Several pieces of the duplicates were produced by pouring the selfcuring acrylic resin into the mold (Fig. 1). They were used to make several neutral zone records for the same patient.

Prior to placing soft wax on the base plate, keels were attached to the plate to maintain the correct vertical dimension. Toyoda type keels $^{22)}$ were employed, which could avoid the undesirable deformation of the soft wax rim often observed in the case of using Lott

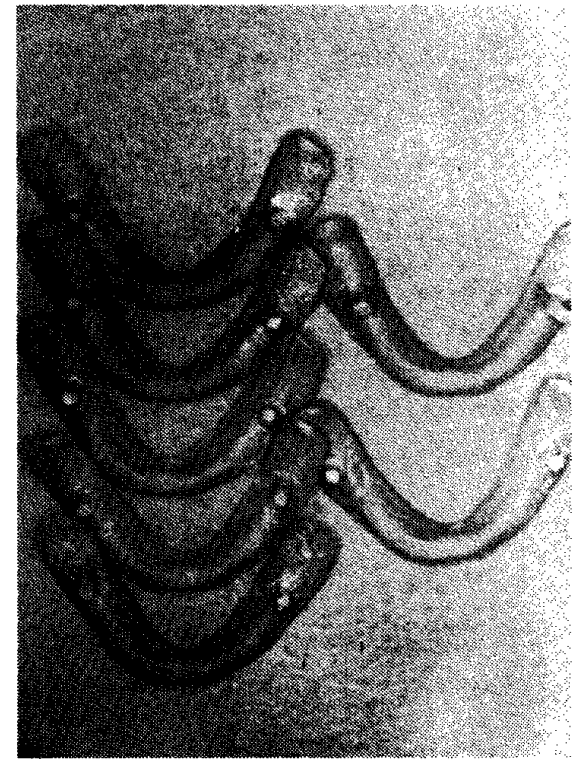

Fig. 1 Duplicates of base plate. type keels. The plate was stored in water bath at $48^{\circ} \mathrm{C}$ for more than 3 minutes to soften the wax uniformly. It was then inserted into the mouth. The patient was directed to conduct some functional movements necessary for recording the neutral zone such as swallowing, pursing lips as in sucking, pulling labial angles to both sides, and speaking. The period for this procedure was limited to approximately 2 minutes, because the longer period of continuous functioning might cause erroneous records due to some unnatural movements of the tongue and because the wax might lose its easy plasticity. Any excessive soft wax above the height of the esimated occlusal plane was removed with a knife. After having been softened again in water 
bath, the wax rim was placed back into the mouth and the recording was resumed. A series of the procedure of softening the wax and recording the neutral zone was continued until the record was assessed to be in its best situation. Principal checking items were: stability of the plate in speaking loudly as well as in opening the mouth widely; status of the tongue space; relationship of the wax rim with the resting tongue, cheeks and lips; appearance of the cheeks and lips; and pronunciation and comfort of the patient.

Projected contour of the completed neutral zone record on the occlusal plane and the alveolar crest of the ridge (Fig. 2) were traced on a paper in a manner shown in Fig. 3. Relative positions of the labial, buccal and lingual margins of the neutral

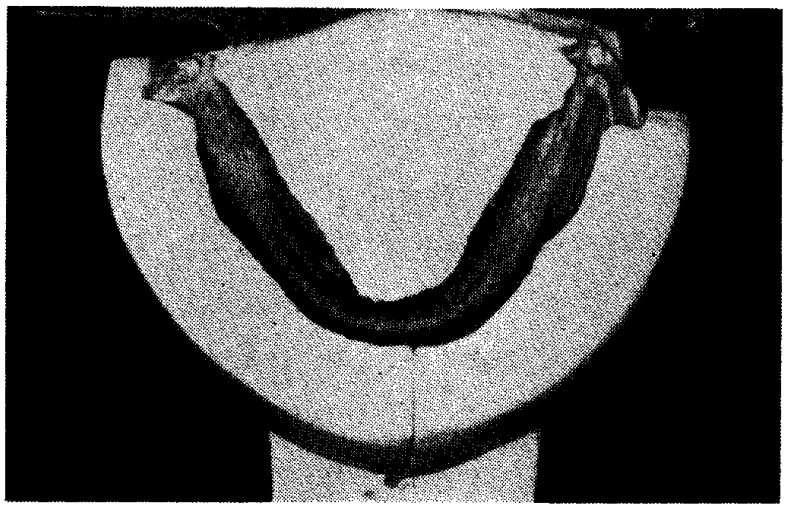

Fig. 2 Completed neutral zone record.

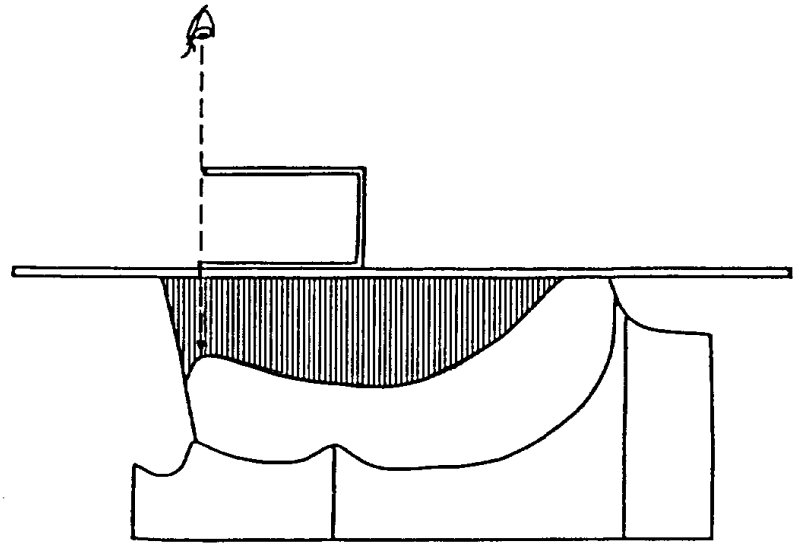

Fig. 3 Schematic illustration of the method for tracing the projected contour of the neutral zone and the alveolar crest of the ridge on the occlusal plane.

zone and the alveolar crest were measured on the traced figures. From these values labio-lingual or bucco-lingual width and mid-point were calculated. The area corresponding to the tongue space on the occlusal plane and other comparable areas were also measured using a planimeter. Two to five records of the neutral zone were made repeatedly under the same condition, and the differences by repetition in those values were statistically analysed by t-test. The experiments were carried out for fifteen edentulous patients ( 6 males and 9 females).

Furthermore, the neutral zone records were constructed under the altered recording condition in several cases, by another practitioner, raising the vertical dimension of occlusion by $5 \mathrm{~mm}$ at the anterior, utilizing the accustomed denture in maxilla, or shifting the positions of the keels to the buccal side. They were independently compared with those under the normal condition.

\section{Results}

Horizontal section of an obtained neutral zone on the hypothetical occlusal plane was drawn by chain line in Fig. 4 . The alveolar ridge crest of the mandible was also 


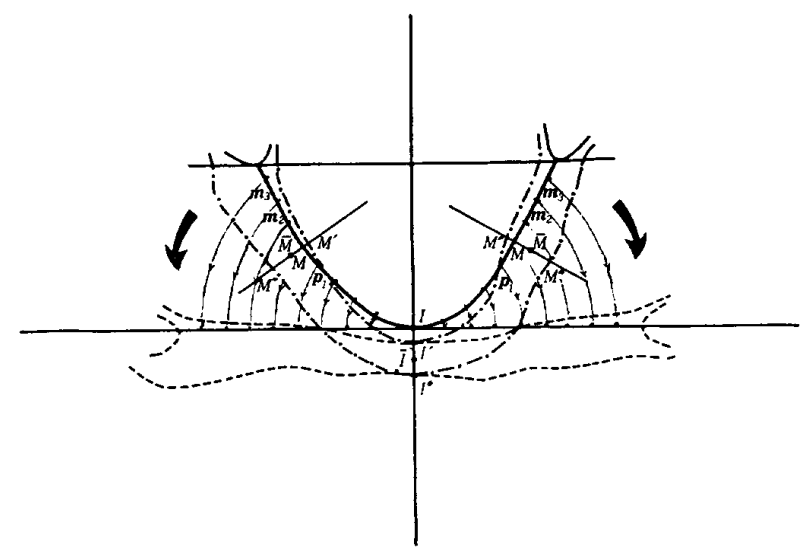

Fig. 4 An example of obtained neutral zone record (chain line) and converted one (broken line) on the median line-alveolar crest rectangular coordinate. shown by solid line. Median line was approximated by the bisector of the straight line between the right and left retromolar pads, and the symbol I represents the intersecting point of the median line with the alveolar crest. In order to make it easier to interpret the data, the crest and the median line were converted into a rectangular coordinate and the neutral zone was replaced on the new coordinate as shown by broken line in Fig. 4. The middle point on the crest between the point I and the anterior margin of the retromolar pad was indicated by $M$, which seemed to correspond approximately with the position of the second premolar or the first molar of the denture. Then the points $m_{1}, m_{2}$ and $\mathrm{m}_{3}$ were set up at $5 \mathrm{~mm}$ intervals distally from the point $M$, and $\mathrm{p}_{1}, \mathrm{p}_{2}, \mathrm{p}_{3}$ and $\mathrm{p}_{4}$ mesially. The lingual and buccal margins of the neutral zone at the reference point $M$ were marked by $M^{\prime}$ and $M^{\prime \prime}$, respectively, and $\bar{M}$ was the mid-point between $M^{\prime}$ and $M^{\prime \prime}$. The same rules were applied correspondingly to other reference points.

Relative positions of these points of the neutral zone were expressed by the distance from the alveolar crest. Recording of the neutral zone was tried two to five times repeatedly for each patient by an experienced practitioner. Tables 1 and 2 show the mean values of the margins at some representative reference points with standard deviations by repetition. Mid-point and width of the neutral zone were calculated from these values at the corresponding reference point and listed in Tables 3 and 4 . Margin lines of the neutral zone for each case were drawn in Figs. 5-1 to 5-4, by plotting $\mathrm{I}^{\prime}, \mathrm{I}^{\prime \prime}, \mathrm{M}^{\prime}, \mathrm{M}^{\prime \prime}$, and others on the alveolar crest-median line rectangular coordinate. The reference point $M$ was settled at a fixed point on the axis of alveolar crest for all the cases, and so were $\mathrm{m}_{1}, \mathrm{p}_{1}$, and others. Center line running on the mid-points was also drawn in the figure with their sample deviations.

Although any of the neutral zone records was clinically judged to be satisfactory in function by the practitioner and the patient, significant differences were statistically recognized in the mid-point and the width of the neutral zone by repetition in each individual patient. Frequency of the record which is significantly different in mid-point from other records obtained for the same patient was $55.4 \%$ in the incisal region and $66.4 \%$ in the molar. While in width, the frequency was $41.8 \%$ in the incisal $49.1 \%$ in the molar.

The mid-point of the neutral zone was located on the labial side of the alveolar crest in the incisal region except for one case as seen in Table 3 and Figs. 5-1 to 
Table 1 Position of the labial, buccal margin of the neutral zone in relation to the alveolar crest.

\begin{tabular}{|c|c|c|c|c|c|c|c|c|}
\hline \multirow{2}{*}{\multicolumn{2}{|c|}{ Cases }} & \multicolumn{7}{|c|}{ labial and buccal margin $(\mathrm{mm})$} \\
\hline & & $\mathbf{M}^{\prime \prime}$ & $\mathrm{m}_{1}^{\prime \prime}$ & $\mathrm{m}_{2}^{\prime \prime}$ & $\mathrm{p}_{1}^{\prime \prime}$ & $\mathrm{p}_{3}^{\prime \prime}$ & $\mathrm{p}_{4}^{\prime \prime}$ & $I^{\prime \prime}$ \\
\hline \multirow{2}{*}{1} & $L$ & $5.03 \pm 0.83$ & $4.57 \pm 1.37$ & $4.40 \pm 1.27$ & $5.17 \pm 0.42$ & $4.73 \pm 0.38$ & $4.33 \pm 0.33$ & \multirow{2}{*}{$4.20 \pm 1.87$} \\
\hline & $R$ & $5.20 \pm 0.80$ & $5.00 \pm 0.85$ & $4.33 \pm 1.07$ & $5.27 \pm 0.82$ & $5.33 \pm 0.87$ & $5.40 \pm 1.51$ & \\
\hline \multirow{2}{*}{2} & $L$ & $7.90 \pm 0.45$ & $7.57 \pm 0.54$ & $6.53 \pm 0.56$ & $8.03 \pm 2.06$ & $8.73 \pm 1.01$ & $8.87 \pm 0.33$ & \multirow{2}{*}{$9.27 \pm 0.62$} \\
\hline & $R$ & $7.27 \pm 1.30$ & $7.03 \pm 1.13$ & $7.23 \pm 1.52$ & $8.00 \pm 1.59$ & $9.47 \pm 1.01$ & $9.17 \pm 0.92$ & \\
\hline \multirow{2}{*}{3} & $L$ & $8.05 \pm 1.86$ & $7.23 \pm 1.86$ & $7.25 \pm 1.40$ & $7.20 \pm 1.60$ & $6.03 \pm 0.78$ & $6.13 \pm 0.95$ & \multirow{2}{*}{$8.10 \pm 1.39$} \\
\hline & $R$ & $8.33 \pm 0.88$ & $8.05 \pm 1.13$ & $7.70 \pm 1.82$ & $8.03 \pm 0.74$ & $7.78 \pm 1.52$ & $7.00 \pm 1.57$ & \\
\hline \multirow{2}{*}{4} & $L$ & $6.30 \pm 0.20$ & $7.05 \pm 0.45$ & $7.05 \pm 0.15$ & $6.00 \pm 0.10$ & $5.25 \pm 0.55$ & $5.70 \pm 0.70$ & \multirow{2}{*}{$7.80 \pm 0.20$} \\
\hline & $\boldsymbol{R}$ & $7.60 \pm 0.50$ & $7.60 \pm 0.50$ & $7.05 \pm 0.05$ & $7.45 \pm 0.45$ & $8.45 \pm 0.45$ & $8.25 \pm 0.05$ & \\
\hline \multirow{2}{*}{5} & $L$ & $10.55 \pm 0.75$ & $10.90 \pm 0.60$ & $11.10 \pm 0.70$ & $10.50 \pm 0.70$ & $8.30 \pm 0.70$ & $7.40 \pm 0.40$ & \multirow{2}{*}{$7.45 \pm 0.35$} \\
\hline & $R$ & $7.85 \pm 0.35$ & $8.30 \pm 0.40$ & $8.05 \pm 1.05$ & $7.60 \pm 0.20$ & $6.45 \pm 0.95$ & $6.25 \pm 0.75$ & \\
\hline \multirow{2}{*}{6} & $L$ & $9.03 \pm 0.45$ & $8.45 \pm 0.43$ & $7.38 \pm 1.00$ & $8.58 \pm 0.43$ & $8.00 \pm 1.41$ & $7.55 \pm 1.51$ & \multirow{2}{*}{$6.28 \pm 092$} \\
\hline & $R$ & $3.20 \pm 1.78$ & $3.90 \pm 0.95$ & $5.48 \pm 0.67$ & $4.53 \pm 1.53$ & $5.88 \pm 0.49$ & $5.45 \pm 0.43$ & \\
\hline \multirow{2}{*}{7} & $L$ & $4.50 \pm 0.57$ & $4.53 \pm 0.65$ & $4.15 \pm 0.99$ & $4.85 \pm 1.25$ & $5.58 \pm 1.92$ & $6.25 \pm 2.28$ & \multirow{2}{*}{$6.08 \pm 2.03$} \\
\hline & $R$ & $5.43 \pm 0.51$ & $4.70 \pm 0.79$ & $2.85 \pm 0.54$ & $5.70 \pm 0.90$ & $5.60 \pm 1.98$ & $6.08 \pm 1.73$ & \\
\hline \multirow{2}{*}{8} & $L$ & $4.03 \pm 1.34$ & $5.08 \pm 1.56$ & $6.45 \pm 0.85$ & $2.80 \pm 1.34$ & $1.90 \pm 1.41$ & $1.90 \pm 1.34$ & \multirow{2}{*}{$2.53 \pm 1.33$} \\
\hline & $R$ & $3.38 \pm 1.29$ & $4.03 \pm 1.52$ & $4.65 \pm 1.25$ & $2.68 \pm 0.51$ & $2.68 \pm 0.61$ & $1.73 \pm 0.92$ & \\
\hline \multirow{2}{*}{9} & $L$ & $7.63 \pm 0.52$ & $6.70 \pm 0.08$ & $5.63 \pm 0.09$ & $8.10 \pm 0.75$ & $9.17 \pm 0.85$ & $10.57 \pm 0.82$ & \multirow{2}{*}{$10.30 \pm 0.24$} \\
\hline & $R$ & $3.13 \pm 0.66$ & $3.33 \pm 0.31$ & $3.03 \pm 0.95$ & $4.33 \pm 0.68$ & $5.43 \pm 0.74$ & $7.10 \pm 0.59$ & \\
\hline \multirow{2}{*}{10} & $L$ & $6.00 \pm 1.12$ & $5.28 \pm 1.41$ & $5.30 \pm 1.63$ & $6.95 \pm 1.38$ & $11.15 \pm 1.15$ & $10.80 \pm 1.73$ & \multirow{2}{*}{$10.90 \pm 1.69$} \\
\hline & $R$ & $7.35 \pm 0.86$ & $6.73 \pm 0.58$ & $6.40 \pm 0.17$ & $8.35 \pm 1.47$ & $10.68 \pm 2.26$ & $10.90 \pm 1.64$ & \\
\hline \multirow{2}{*}{11} & $L$ & $3.90 \pm 0.90$ & $3.65 \pm 0.65$ & $4.85 \pm 0.15$ & $3.25 \pm 0.85$ & $4.75 \pm 1.05$ & $5.40 \pm 0.90$ & \multirow{2}{*}{$5.95 \pm 0.15$} \\
\hline & $R$ & $3.75 \pm 0.05$ & $5.15 \pm 0.05$ & $5.25 \pm 0.05$ & $3.00 \pm 0.00$ & $3.05 \pm 0.05$ & $3.15 \pm 0.05$ & \\
\hline \multirow{2}{*}{12} & $L$ & $6.00 \pm 0.00$ & $4.50 \pm 0.00$ & $3.75 \pm 0.15$ & $6.85 \pm 0.05$ & $6.80 \pm 0.00$ & $6.35 \pm 0.75$ & \multirow{2}{*}{$6.35 \pm 0.85$} \\
\hline & $R$ & $4.75 \pm 0.25$ & $4.65 \pm 0.25$ & $3.50 \pm 0.00$ & $5.45 \pm 0.35$ & $5.55 \pm 0.35$ & $6.35 \pm 0.55$ & \\
\hline \multirow{2}{*}{13} & $L$ & $7.40 \pm 0.85$ & $7.17 \pm 0.69$ & $6.33 \pm 0.74$ & $7.60 \pm 0.50$ & $7.80 \pm 0.22$ & $7.67 \pm 0.24$ & \multirow{2}{*}{$7.90 \pm 0.08$} \\
\hline & $R$ & $9.17 \pm 0.62$ & $8.50 \pm 0.45$ & $8.00 \pm 0.24$ & $9.53 \pm 0.73$ & $8.67 \pm 0.47$ & $8.70 \pm 0.57$ & \\
\hline 14 & $L$ & $7.00 \pm 1.00$ & $6.80 \pm 0.80$ & $6.30 \pm 0.90$ & $7.25 \pm 0.75$ & $8.45 \pm 0.55$ & $9.05 \pm 0.05$ & +0.40 \\
\hline 14 & $R$ & $7.75 \pm 0.05$ & $7.80 \pm 0.10$ & $8.35 \pm 0.45$ & $7.90 \pm 0.20$ & $8.75 \pm 0.25$ & $9.70 \pm 0.20$ & 10 \\
\hline 15 & $L$ & $5.57 \pm 0.83$ & $5.07 \pm 1.25$ & $4.00 \pm 1.70$ & $6.33 \pm 1.25$ & $8.07 \pm 1.35$ & $8.73 \pm 1.16$ & 9.0 \\
\hline 10 & $R$ & $6.40 \pm 0.29$ & $6.10 \pm 0.22$ & $4.73 \pm 0.76$ & $5.87 \pm 0.17$ & $7.03 \pm 0.74$ & $7.70 \pm 0.36$ & \\
\hline & $L$ & $6.63 \pm 0.78$ & $6.04 \pm 0.82$ & $6.03 \pm 0.82$ & $6.63 \pm 0.72$ & $6.98 \pm 0.95$ & $7.11 \pm 0.90$ & 37 \\
\hline & $R$ & $6.04 \pm 0.68$ & $6.06 \pm 0.62$ & $5.78 \pm 0.71$ & $6.25 \pm 0.69$ & $6.58 \pm 0.85$ & $6.92 \pm 0.79$ & -0.01 \\
\hline
\end{tabular}

Positive value : distance from the crest to labial or buccal side 
Table 2 Position of the lingual margin of the neutral zone in relation to the alveolar crest.

\begin{tabular}{|c|c|c|c|c|c|c|c|c|}
\hline \multirow{2}{*}{\multicolumn{2}{|c|}{ Cases }} & \multicolumn{7}{|c|}{ lingual margin $(\mathrm{mm})$} \\
\hline & & $M^{\prime}$ & $\mathrm{m}_{1}^{\prime}$ & $\mathrm{m}_{2}^{\prime}$ & $\mathrm{p}_{1}^{\prime}$ & $\mathrm{p}_{3}{ }^{\prime}$ & $4^{\prime}$ & $I^{\prime}$ \\
\hline \multirow{2}{*}{1} & $L$ & $6.20 \pm 1.22$ & $5.57 \pm 1.73$ & $4.60 \pm 2.43$ & $5.90 \pm 1.14$ & & & \multirow{2}{*}{$-0.07 \pm 2.34$} \\
\hline & $R$ & \pm 1.15 & $5.43 \pm 1.61$ & $4.93 \pm 1.54$ & $5.70 \pm 1.34$ & $2.40 \pm 1.42$ & $1.27 \pm 1.92$ & \\
\hline \multirow{2}{*}{2} & $L$ & $0.10 \pm 0.57$ & $1.23 \pm 0.66$ & $1.97 \pm 0.40$ & $-0.43 \pm 0.42$ & & $-2.87 \pm 0.78$ & \multirow{2}{*}{$-3.33 \pm 0.47$} \\
\hline & $R$ & $1.67 \pm 0.62$ & $2.20 \pm 0.62$ & $2.07 \pm 0.65$ & $0.80 \pm 0.22$ & $-1.87 \pm 0.17$ & $-2.70 \pm 0.57$ & \\
\hline \multirow{2}{*}{3} & $L$ & $4.75 \pm 1.90$ & $3 \pm 1.94$ & $0 \pm 1.80$ & 37 & 64 & $4.48 \pm 1.26$ & \multirow{2}{*}{$2.68 \pm 0.26$} \\
\hline & $R$ & $4.10 \pm 1.24$ & $4.60 \pm 0.60$ & $4.10 \pm 0.70$ & $4.25 \pm 1.11$ & $2.25 \pm 0.90$ & $1.65 \pm 0.74$ & \\
\hline \multirow{2}{*}{4} & $L$ & $0.95 \pm 0.05$ & $0.64 \pm 0.35$ & $-0.60 \pm 1.20$ & 45 & & 0.60 & \multirow{2}{*}{$-3.30 \pm 0.80$} \\
\hline & $R$ & $-0.40 \pm 0.20$ & $-0.20 \pm 0.40$ & $-1.05 \pm 0.35$ & $-1.30 \pm 0.60$ & $-2.05 \pm 0.35$ & $-3.05 \pm 0.25$ & \\
\hline \multirow{2}{*}{5} & $L$ & $0.90 \pm 0.90$ & $0.80 \pm 1.30$ & $0.65 \pm 1.65$ & 75 & & & \multirow{2}{*}{$1.00 \pm 2.10$} \\
\hline & $R$ & $3.80 \pm 0.30$ & $3.35 \pm 0.25$ & $2.75 \pm 0.05$ & $3.20 \pm 0.60$ & $1.65 \pm 1.55$ & $1.60 \pm 1.60$ & \\
\hline \multirow{2}{*}{6} & $L$ & $0.93 \pm 0.73$ & $1.10 \pm 0.77$ & & $0.0 \pm$ & & 43 & \multirow{2}{*}{$-0.50 \pm 1.30$} \\
\hline & $R$ & $6.38 \pm 0.87$ & $5.75 \pm 1.10$ & $3.95 \pm 1.23$ & $4.45 \pm 0.71$ & $2.00 \pm 1.16$ & $1.60 \pm 1.21$ & \\
\hline \multirow{2}{*}{7} & $L$ & $4.48 \pm 2.19$ & $4.00 \pm 1.82$ & $5 \pm 1.65$ & $8 \pm 2.80$ & & & \multirow{2}{*}{$1.93 \pm 2.91$} \\
\hline & $R$ & $2.63 \pm 1.90$ & $2.93 \pm 1.46$ & $3.50 \pm 1.07$ & $3.00 \pm 2.44$ & $1.80 \pm 3.84$ & $0.23 \pm 3.60$ & \\
\hline \multirow{2}{*}{8} & $L$ & $4.23 \pm 0.84$ & $4.82 \pm 0.24$ & $5.18 \pm 0.61$ & & & & \multirow{2}{*}{$2.90 \pm 0.95$} \\
\hline & $R$ & $5.33 \pm 1.27$ & $5.35 \pm 1.36$ & $5.08 \pm 1.27$ & $4.85 \pm 1.14$ & $3.50 \pm 0.87$ & $3.88 \pm$ & \\
\hline \multirow{2}{*}{9} & $L$ & $0.80 \pm 0.70$ & $1.33 \pm 0.97$ & $2.10 \pm 0.70$ & $.00 \pm 0.45$ & -2.3 & & \multirow{2}{*}{$-2.37 \pm 1.44$} \\
\hline & $R$ & $3.17 \pm 1.25$ & $3.33 \pm 1.36$ & $3.53 \pm 1.11$ & $2.73 \pm 0.97$ & $1.83 \pm 1.25$ & $0.33 \pm 0.24$ & \\
\hline \multirow{2}{*}{10} & $L$ & $2.60 \pm 0.52$ & $3.53 \pm 0.60$ & & $0.40 \pm 1.14$ & & $8 \pm 1.60$ & \multirow{2}{*}{$-4.85 \pm 1.76$} \\
\hline & $R$ & $1.05 \pm 0.84$ & $2.28 \pm 1.00$ & $2.38 \pm 0.96$ & $-0.55 \pm 1.56$ & $-4.48 \pm 2.32$ & $-4.85 \pm 1.61$ & \\
\hline \multirow{2}{*}{11} & $L$ & $4.85 \pm 0.25$ & $4.15 \pm 0.25$ & $1.35 \pm 0.45$ & $3.00 \pm 0.10$ & $1.05 \pm 0.15$ & $0.20 \pm 1.10$ & \multirow{2}{*}{$-2.55 \pm 0.55$} \\
\hline & $R$ & $0.70 \pm 0.00$ & $1.45 \pm 0.05$ & $1.95 \pm 0.05$ & $0.00 \pm 0.00$ & $0.10 \pm 0.00$ & $-0.20 \pm 0.00$ & \\
\hline \multirow{2}{*}{12} & $L$ & $0.90 \pm 0.10$ & $1.80 \pm 0.00$ & $5 \pm 0.05$ & $0.50 \pm 0.50$ & $-1.90 \pm 0.10$ & $-2.70 \pm 0.20$ & \multirow{2}{*}{$-3.35 \pm 0.85$} \\
\hline & $R$ & $-0.45 \pm 0.45$ & $1.00 \pm 0.30$ & $1.25 \pm 1.05$ & $-0.95 \pm 0.05$ & $-2.60 \pm 1.10$ & $-3.65 \pm 0.85$ & \\
\hline \multirow{2}{*}{13} & $L$ & $3.00 \pm 0.57$ & $2.87 \pm 0.74$ & $2.87 \pm 1.03$ & $2.90 \pm 0.22$ & $0.57 \pm 0.60$ & $-0.33 \pm 1.18$ & \multirow{2}{*}{$-4.30 \pm 0.50$} \\
\hline & $R$ & $-0.60 \pm 0.70$ & $-0.10 \pm 0.29$ & $0.47 \pm 0.05$ & $-0.80 \pm 1.35$ & $-3.67 \pm 1.25$ & $-4.87 \pm 0.98$ & \\
\hline 14 & $L$ & $2.80 \pm 0.30$ & $3.85 \pm 0.35$ & $3.90 \pm 0.30$ & $1.05 \pm 0.25$ & $-2.45 \pm 0.55$ & $-4.55 \pm 0.65$ & 0 \\
\hline 14 & $R$ & $0.90 \pm 0.90$ & $1.20 \pm 0.70$ & $1.25 \pm 0.75$ & $0.20 \pm 0.50$ & $-2.10 \pm 0.20$ & $-4.80 \pm 1.10$ & \\
\hline 15 & $L$ & $4.50 \pm 1.04$ & $3.73 \pm 0.83$ & $3.13 \pm 0.98$ & $3.20 \pm 1.10$ & $-1.63 \pm 1.48$ & $-3.83 \pm 1.43$ & -5 \\
\hline & $R$ & $3.17 \pm 1.70$ & $3.07 \pm 1.51$ & $2.73 \pm 0.04$ & $2.87 \pm 1.56$ & $-0.80 \pm 0.83$ & $-2.10 \pm 0.62$ & \\
\hline & $L$ & $2.80 \pm 0.79$ & $2.95 \pm 0.84$ & $2.58 \pm 1.00$ & $2.22 \pm 0.91$ & $0.37 \pm 0.99$ & $-0.67 \pm 1.18$ & \\
\hline & $R$ & $2.49 \pm 0.89$ & $2.78 \pm 0.84$ & $2.59 \pm 0.72$ & $0.50 \pm 0.94$ & $-0.14 \pm 1.15$ & $-1.04 \pm 1.07$ & \\
\hline
\end{tabular}

Positive value: distance from the crest to ligual side

Negative value : distance from the crest to labial or buccal side 
Table 3 Position of the mid-point of the neutral zone in relation to the alveolar crest.

\begin{tabular}{|c|c|c|c|c|c|c|c|c|}
\hline \multirow{2}{*}{\multicolumn{2}{|c|}{ Cases }} & \multirow[b]{2}{*}{$\overline{\mathbf{M}}$} & \multirow[b]{2}{*}{$\mathrm{m}_{1}$} & \multicolumn{3}{|c|}{ mid point $(\mathrm{mm})$} & \multirow{2}{*}{$\overline{\mathrm{p}}_{4}$} & \multirow[b]{2}{*}{$\overline{\mathrm{I}}$} \\
\hline & & & & $\overline{\mathrm{m}}_{2}$ & $\overline{\mathbf{p}}_{1}$ & $\overline{\mathrm{p}}_{3}$ & & \\
\hline \multirow{2}{*}{1} & $L$ & $-0.63 \pm 0.62$ & $-0.53 \pm 1.04$ & $-0.10 \pm 1.24$ & $-0.33 \pm 0.52$ & $0.23 \pm 0.45$ & $0.53 \pm 0.59$ & \multirow{2}{*}{$1.33 \pm 1.16$} \\
\hline & $R$ & $-0.33 \pm 0.83$ & $-0.30 \pm 1.28$ & $-0.30 \pm 1.18$ & $-0.20 \pm 0.82$ & $1.50 \pm 1.10$ & $2.10 \pm 1.69$ & \\
\hline \multirow{2}{*}{2} & $L$ & $3.93 \pm 0.25$ & $3.20 \pm 0.37$ & $2.30 \pm 0.16$ & $4.27 \pm 0.31$ & $5.27 \pm 0.78$ & $5.57 \pm 0.31$ & \multirow{2}{*}{$6.63 \pm 0.54$} \\
\hline & $R$ & $2.83 \pm 0.95$ & $2.40 \pm 0.91$ & $2.50 \pm 0.22$ & $3.60 \pm 0.83$ & $5.70 \pm 0.43$ & $5.93 \pm 0.45$ & \\
\hline \multirow{2}{*}{3} & $L$ & $1.68 \pm 0.83$ & $1.23 \pm 0.77$ & $1.80 \pm 0.65$ & $1.13 \pm 0.91$ & $0.55 \pm 0.50$ & $0.85 \pm 0.41$ & \multirow{2}{*}{$2.70 \pm 0.60$} \\
\hline & $R$ & $2.15 \pm 0.65$ & $1.75 \pm 0.63$ & $1.83 \pm 1.18$ & $1.90 \pm 0.51$ & $2.80 \pm 1.14$ & $3.10 \pm 1.07$ & \\
\hline \multirow{2}{*}{4} & $L$ & $2.95 \pm 0.15$ & $3.20 \pm 0.40$ & $4.10 \pm 0.90$ & $2.30 \pm 0.20$ & $1.40 \pm 0.50$ & $2.55 \pm 0.55$ & \multirow{2}{*}{$5.60 \pm 0.30$} \\
\hline & $R$ & $4.05 \pm 0.35$ & $4.15 \pm 0.25$ & $4.05 \pm 0.15$ & $4.40 \pm 0.10$ & $5.30 \pm 0.40$ & $5.65 \pm 0.15$ & \\
\hline \multirow{2}{*}{5} & $L$ & $4.85 \pm 0.85$ & $5.05 \pm 0.95$ & $5.25 \pm 1.15$ & $4.75 \pm 0.75$ & $4.00 \pm 0.90$ & $3.70 \pm 0.80$ & \multirow{2}{*}{$3.25 \pm 1.25$} \\
\hline & $R$ & $2.05 \pm 0.35$ & $2.50 \pm 0.30$ & $2.70 \pm 0.50$ & $2.20 \pm 0.40$ & $2.45 \pm 1.25$ & $2.35 \pm 1.15$ & \\
\hline \multirow{2}{*}{6} & $L$ & $4.05 \pm 0.52$ & $3.70 \pm 0.59$ & $3.02 \pm 0.87$ & $4.13 \pm 0.60$ & $4.40 \pm 1.27$ & $4.30 \pm 1.43$ & \multirow{2}{*}{$3.43 \pm 1.10$} \\
\hline & $R$ & $-1.63 \pm 0.96$ & $-0.95 \pm 0.39$ & $0.78 \pm 0.89$ & $0.05 \pm 0.92$ & $1.98 \pm 0.80$ & $1.95 \pm 0.80$ & \\
\hline \multirow{2}{*}{7} & $L$ & $0.03 \pm 1.29$ & $0.28 \pm 1.02$ & $0.55 \pm 1.05$ & $0.60 \pm 1.98$ & $2.18 \pm 2.43$ & $2.90 \pm 2.60$ & \multirow{2}{*}{$3.38 \pm 2.72$} \\
\hline & $R$ & $1.43 \pm 1.09$ & $0.90 \pm 0.97$ & $-0.30 \pm 0.70$ & $1.38 \pm 1.58$ & $1.90 \pm 2.87$ & $2.95 \pm 2.66$ & \\
\hline \multirow{2}{*}{8} & $L$ & $0.15 \pm 0.50$ & $0.13 \pm 0.80$ & $0.65 \pm 0.63$ & $-0.63 \pm 1.05$ & $-1.95 \pm 1.72$ & $-1.65 \pm 1.57$ & \multirow{2}{*}{$-0.18 \pm 1.14$} \\
\hline & $R$ & $-0.95 \pm 1.25$ & $-0.93 \pm 1.21$ & $-0.18 \pm 1.22$ & $-1.08 \pm 0.75$ & $-0.70 \pm 0.73$ & $-1.08 \pm 0.83$ & \\
\hline \multirow{2}{*}{9} & $L$ & $3.43 \pm 0.40$ & $2.70 \pm 0.51$ & $1.80 \pm 0.33$ & $3.57 \pm 0.34$ & $5.73 \pm 0.90$ & $0.83 \pm 0.95$ & \multirow{2}{*}{$6.37 \pm 0.82$} \\
\hline & $\boldsymbol{R}$ & $0.00 \pm 0.37$ & $0.03 \pm 0.66$ & $0.23 \pm 0.69$ & $0.82 \pm 0.18$ & $1.80 \pm 0.99$ & $3.40 \pm 0.43$ & \\
\hline \multirow{2}{*}{10} & $L$ & $1.70 \pm 0.60$ & $0.85 \pm 0.53$ & $1.05 \pm 0.86$ & $3.30 \pm 1.22$ & $6.85 \pm 1.49$ & $7.80 \pm 1.63$ & \multirow{2}{*}{$7.78 \pm 1.55$} \\
\hline & $\boldsymbol{R}$ & $3.18 \pm 0.78$ & $2.15 \pm 0.59$ & $1.85 \pm 0.79$ & $4.48 \pm 1.46$ & $7.60 \pm 2.19$ & $7.90 \pm 1.48$ & \\
\hline \multirow{2}{*}{11} & $L$ & $-0.70 \pm 0.10$ & $-0.25 \pm 0.45$ & $1.75 \pm 0.15$ & $0.15 \pm 0.45$ & $1.85 \pm 0.45$ & $2.60 \pm 0.10$ & \multirow{2}{*}{$4.20 \pm 0.40$} \\
\hline & $\boldsymbol{R}$ & $1.50 \pm 0.00$ & $1.85 \pm 0.05$ & $1.65 \pm 0.05$ & $1.50 \pm 0.00$ & $1.50 \pm 0.00$ & $1.70 \pm 0.00$ & \\
\hline 12 & $L$ & $2.55 \pm 0.05$ & $1.40 \pm 0.00$ & $0.85 \pm 0.05$ & $3.20 \pm 0.20$ & $4.35 \pm 0.05$ & $4.55 \pm 0.45$ & -0.85 \\
\hline & $R$ & $2.60 \pm 0.10$ & $1.85 \pm 0.25$ & $1.15 \pm 0.55$ & $3.20 \pm 0.20$ & $4.10 \pm 0.70$ & $5.00 \pm 0.70$ & \\
\hline 13 & $L$ & $2.23 \pm 0.73$ & $2.17 \pm 0.71$ & $1.40 \pm 0.73$ & $2.37 \pm 0.34$ & $3.47 \pm 0.33$ & $4.00 \pm 0.71$ & $6.10+0.22$ \\
\hline & $R$ & $5.23 \pm 0.17$ & $4.30 \pm 0.37$ & $3.77 \pm 0.12$ & $5.17 \pm 0.31$ & $6.17 \pm 0.85$ & $6.80 \pm 0.78$ & \\
\hline 14 & $L$ & $2.15 \pm 0.65$ & $1.50 \pm 0.60$ & $1.20 \pm 0.60$ & $3.10 \pm 0.50$ & $5.70 \pm 0.80$ & $6.80 \pm 0.30$ & +0.65 \\
\hline & $R$ & $3.45 \pm 0.45$ & $3.30 \pm 0.40$ & $3.55 \pm 0.15$ & $3.85 \pm 0.15$ & $5.45 \pm 0.05$ & $7.25 \pm 0.65$ & \\
\hline 15 & $L$ & $0.73 \pm 0.61$ & $0.70 \pm 0.59$ & $0.43 \pm 0.58$ & $1.60 \pm 1.00$ & $4.87 \pm 1.39$ & $6.30 \pm 1.21$ & +0.97 \\
\hline & $R$ & $1.63 \pm 0.96$ & $1.57 \pm 0.74$ & $1.03 \pm 0.52$ & $1.50 \pm 0.83$ & $3.93 \pm 0.69$ & $4.90 \pm 0.36$ & \\
\hline & $L$ & $1.94 \pm 0.54$ & $1.69 \pm 0.62$ & $1.74 \pm 0.66$ & $2.23 \pm 0.69$ & $3.26 \pm 0.93$ & $3.84 \pm 0.91$ & 4 \\
\hline & $R$ & $1.81 \pm 0.62$ & $1.64 \pm 0.60$ & $1.62 \pm 0.59$ & $2.18 \pm 0.60$ & $3.43 \pm 0.95$ & $3.99 \pm 0.88$ & \\
\hline
\end{tabular}

Positive value : distance from the crest to labial or buccal side

Negative value: distance from the crest to lingual side 
Table 4 Width of the neutral zone.

\begin{tabular}{|c|c|c|c|c|c|c|c|c|}
\hline \multirow{2}{*}{\multicolumn{2}{|c|}{ Cases }} & \multicolumn{7}{|c|}{ width $(\mathrm{mm})$} \\
\hline & & $\mathrm{M}^{\prime}-\mathrm{M}^{\prime \prime}$ & $\mathrm{m}_{1}^{\prime}-\mathrm{m}_{1}^{\prime \prime}$ & $\mathrm{m}_{2}^{\prime}-\mathrm{m}_{2}^{\prime \prime}$ & $\mathrm{p}_{1}^{\prime}-\mathrm{p}_{1}^{\prime \prime}$ & $\mathrm{p}_{3}{ }^{\prime}-\mathrm{p}_{3}^{\prime \prime}$ & $\mathrm{p}_{4}^{\prime}-\mathrm{p}_{4}^{\prime \prime}$ & $I^{\prime}-I^{\prime \prime}$ \\
\hline \multirow{2}{*}{1} & $L$ & $11.23 \pm 1.61$ & $10.07 \pm 2.22$ & $9.00 \pm 3.00$ & $11.07 \pm 1.33$ & $9.07 \pm 0.49$ & $7.67 \pm 0.54$ & \multirow{2}{*}{$4.13 \pm 1.27$} \\
\hline & $R$ & $11.13 \pm 1.16$ & $10.23 \pm 0.98$ & $9.23 \pm 1.25$ & $10.97 \pm 1.52$ & $7.73 \pm 0.66$ & $6.67 \pm 0.48$ & \\
\hline \multirow{2}{*}{2} & $L$ & $8.00 \pm 0.93$ & $8.80 \pm 0.94$ & $8.50 \pm 0.90$ & $7.60 \pm 0.22$ & $7.00 \pm 0.62$ & $6.00 \pm 0.78$ & \multirow{2}{*}{$5.93 \pm 1.09$} \\
\hline & $R$ & $0.93 \pm 0.87$ & $9.13 \pm 0.73$ & $8.43 \pm 0.71$ & $8.80 \pm 1.56$ & $7.60 \pm 1.13$ & $6.47 \pm 1.24$ & \\
\hline \multirow{2}{*}{3} & $L$ & $12.80 \pm 3.35$ & $12.05 \pm 3.49$ & $11.05 \pm 2.95$ & $12.20 \pm 3.62$ & $11.00 \pm 2.38$ & $10.60 \pm 2.09$ & \multirow{2}{*}{$2.68 \pm 0.26$} \\
\hline & $R$ & $12.43 \pm 173$ & $12.65 \pm 1.28$ & $11.80 \pm 1.40$ & $12.28 \pm 1.57$ & $10.03 \pm 0.97$ & $9.45 \pm 1.23$ & \\
\hline \multirow{2}{*}{4} & $L$ & $7.75 \pm 0.15$ & $7.70 \pm 0.10$ & $6.95 \pm 0.55$ & $7.45 \pm 0.55$ & $7.80 \pm 0.10$ & $6.30 \pm 0.30$ & \multirow{2}{*}{$4.50 \pm 1.00$} \\
\hline & $R$ & $7.20 \pm 0.30$ & $7.40 \pm 0.10$ & $6.00 \pm 0.40$ & $6.15 \pm 1.05$ & $6.40 \pm 0.10$ & $5.20 \pm 0.20$ & \\
\hline \multirow{2}{*}{5} & $L$ & $11.45 \pm 0.15$ & $17.70 \pm 0.70$ & $11.75 \pm 0.95$ & $11.55 \pm 0.05$ & $8.70 \pm 0.40$ & $7.45 \pm 0.75$ & \multirow{2}{*}{$8.45 \pm 1.75$} \\
\hline & $R$ & $11.65 \pm 0.05$ & $11.65 \pm 0.15$ & $10.80 \pm 1.10$ & $10.80 \pm 0.40$ & $8.10 \pm 0.60$ & $7.85 \pm 0.85$ & \\
\hline \multirow{2}{*}{6} & $L$ & $9.95 \pm 0.62$ & $9.55 \pm 0.47$ & $8.75 \pm 0.45$ & $8.98 \pm 0.79$ & $7.25 \pm 0.70$ & $6.53 \pm 0.63$ & \multirow{2}{*}{$5.78 \pm 0.50$} \\
\hline & $R$ & $9.58 \pm 2.01$ & $9.31 \pm 1.77$ & $9.43 \pm 0.93$ & $8.98 \pm 1.56$ & $7.88 \pm 0.83$ & $7.05 \pm 0.92$ & \\
\hline \multirow{2}{*}{7} & $L$ & $8.95 \pm 1.85$ & $8.53 \pm 1.85$ & $7.20 \pm 1.71$ & $8.53 \pm 1.89$ & $6.83 \pm 1.22$ & $6.23 \pm 1.42$ & \multirow{2}{*}{$5.40 \pm 1.46$} \\
\hline & $R$ & $8.05 \pm 1.68$ & $7.63 \pm 1.37$ & $6.35 \pm 0.99$ & $8.70 \pm 1.83$ & $7.40 \pm 2.11$ & $6.30 \pm 1.88$ & \\
\hline \multirow{2}{*}{8} & $L$ & $8.18 \pm 1.89$ & $9.93 \pm 1.53$ & $11.63 \pm 0.71$ & $6.95 \pm 1.31$ & $6.28 \pm 0.84$ & $5.73 \pm 0.94$ & \multirow{2}{*}{$5.43 \pm 0.38$} \\
\hline & $R$ & $8.70 \pm 0.53$ & $9.34 \pm 0.77$ & $9.73 \pm 0.48$ & $7.53 \pm 0.89$ & $5.73 \pm 0.94$ & $5.60 \pm 0.42$ & \\
\hline \multirow{2}{*}{9} & $L$ & $8.43 \pm 0.91$ & $8.03 \pm 0.97$ & $7.73 \pm 0.71$ & $9.10 \pm 1.04$ & $6.85 \pm 0.09$ & $6.87 \pm 0.80$ & \multirow{2}{*}{$7.53 \pm 1.15$} \\
\hline & $R$ & $6.30 \pm 1.84$ & $6.67 \pm 1.47$ & $6.57 \pm 1.53$ & $7.07 \pm 1.65$ & $7.27 \pm 0.52$ & $7.43 \pm 0.37$ & \\
\hline \multirow{2}{*}{10} & $L$ & $8.60 \pm 1.26$ & $8.80 \pm 1.93$ & $8.50 \pm 2.07$ & $7.35 \pm 0.54$ & $5.70 \pm 0.93$ & $6.03 \pm 0.84$ & \multirow{2}{*}{$5.80 \pm 1.42$} \\
\hline & $R$ & $8.40 \pm 0.70$ & $9.00 \pm 1.20$ & $8.38 \pm 0.36$ & $7.80 \pm 0.64$ & $6.20 \pm 1.49$ & $6.05 \pm 1.21$ & \\
\hline \multirow{2}{*}{11} & $L$ & $8.75 \pm 1.15$ & $7.80 \pm 0.40$ & $6.20 \pm 0.60$ & $6.25 \pm 0.75$ & $5.80 \pm 1.20$ & $5.60 \pm 2.00$ & \multirow{2}{*}{$3.40 \pm 0.40$} \\
\hline & $R$ & $4.40 \pm 0.00$ & $6.60 \pm 0.00$ & $7.20 \pm 0.00$ & $3.00 \pm 0.00$ & $3.15 \pm 0.05$ & $2.95 \pm 0.05$ & \\
\hline \multirow{2}{*}{12} & $L$ & $6.90 \pm 0.10$ & $6.30 \pm 0.00$ & $5.80 \pm 0.20$ & $7.35 \pm 0.55$ & $4.90 \pm 0.10$ & $3.65 \pm 0.55$ & \multirow{2}{*}{$3.00 \pm 0.00$} \\
\hline & $R$ & $4.30 \pm 0.70$ & $5.65 \pm 0.05$ & $4.75 \pm 1.05$ & $4.50 \pm 0.30$ & $2.95 \pm 0.75$ & $2.70 \pm 0.30$ & \\
\hline \multirow{2}{*}{13} & $L$ & $10.40 \pm 0.27$ & $10.03 \pm 0.24$ & $9.20 \pm 0.43$ & $10.50 \pm 0.37$ & $8.37 \pm 0.53$ & $7.33 \pm 0.94$ & \multirow{2}{*}{$3.60 \pm 0.57$} \\
\hline & $R$ & $8.57 \pm 1.11$ & $8.40 \pm 0.16$ & $8.47 \pm 0.25$ & $8.73 \pm 2.08$ & $5.00 \pm 0.82$ & $3.83 \pm 0.42$ & \\
\hline 14 & $L$ & $9.80 \pm 0.70$ & $10.65 \pm 0.45$ & $10.20 \pm 0.60$ & $8.30 \pm 0.50$ & $6.67 \pm 0.47$ & $4.50 \pm 0.70$ & \\
\hline 14 & $R$ & $8.65 \pm 0.95$ & $9.00 \pm 0.60$ & $9.60 \pm 1.20$ & $8.10 \pm 0.70$ & $6.65 \pm 0.45$ & $4.90 \pm 0.90$ & \\
\hline 15 & $L$ & $9.73 \pm 1.38$ & $8.80 \pm 1.77$ & $7.13 \pm 2.53$ & $9.53 \pm 1.25$ & $6.43 \pm 0.21$ & $4.90 \pm 1.04$ & \\
\hline 10 & $R$ & $9.57 \pm 1.45$ & $9.17 \pm 1.49$ & $7.47 \pm 2.13$ & $8.73 \pm 1.48$ & $6.23 \pm 0.82$ & $5.60 \pm 0.71$ & \\
\hline & $L$ & $9.40 \pm 1.09$ & $9.25 \pm 1.14$ & $8.64 \pm 1.22$ & $8.85 \pm 0.98$ & $7.34 \pm 0.67$ & $6.36 \pm 0.95$ & \\
\hline & $R$ & $8.52 \pm 1.01$ & $8.79 \pm 0.81$ & $8.57 \pm 0.92$ & $8.14 \pm 1.15$ & $6.55 \pm 0.82$ & $5.87 \pm 0.75$ & \\
\hline
\end{tabular}



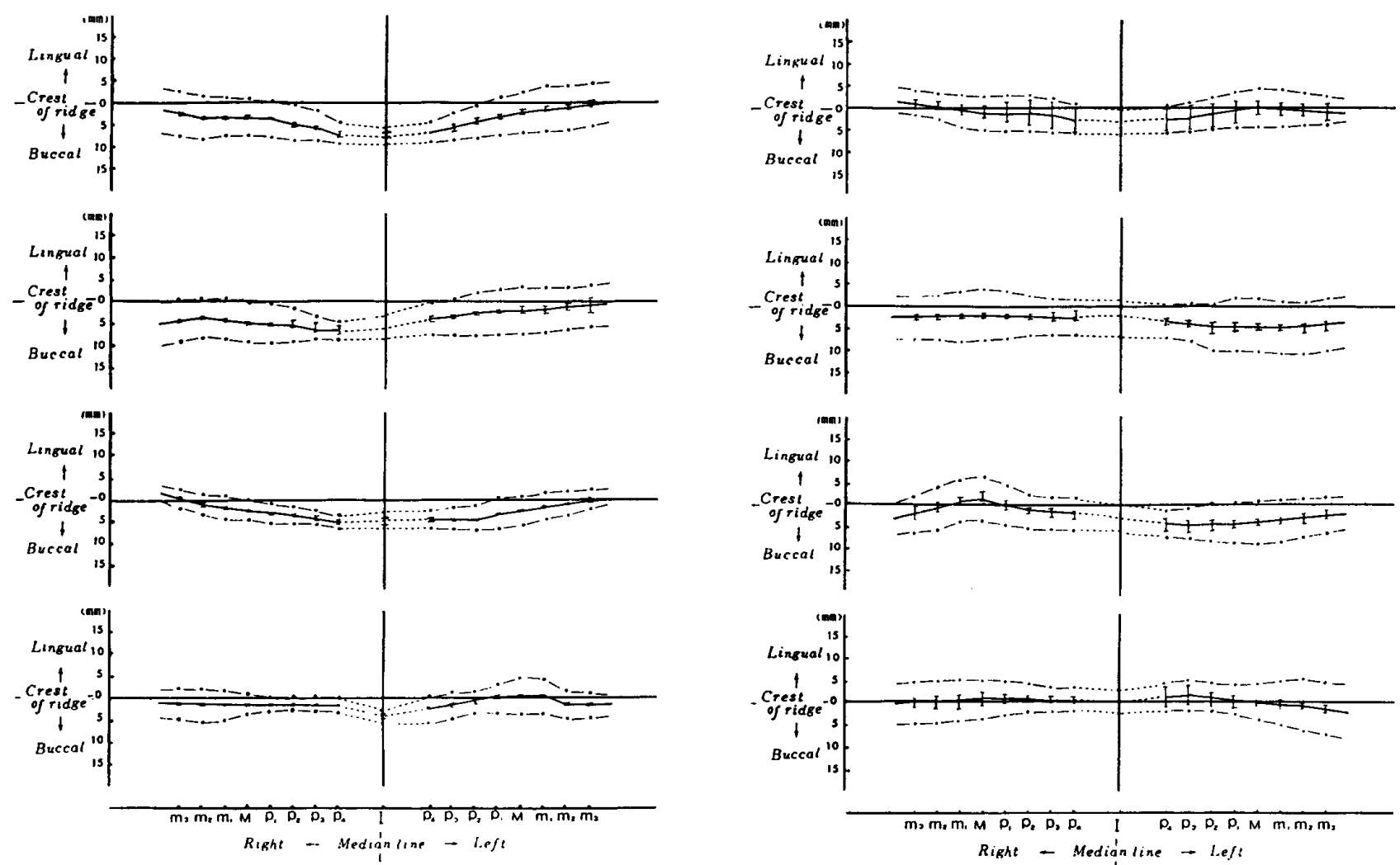

Fig. 5-1 Neutral zone (chain line) and its center line (solid line) for each case.

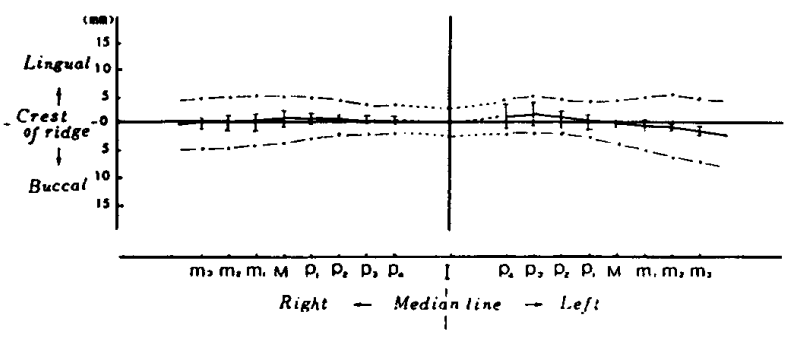

Fig. 5 - 3 Neutral zone (chain line) and its center line (solid line) for each case.
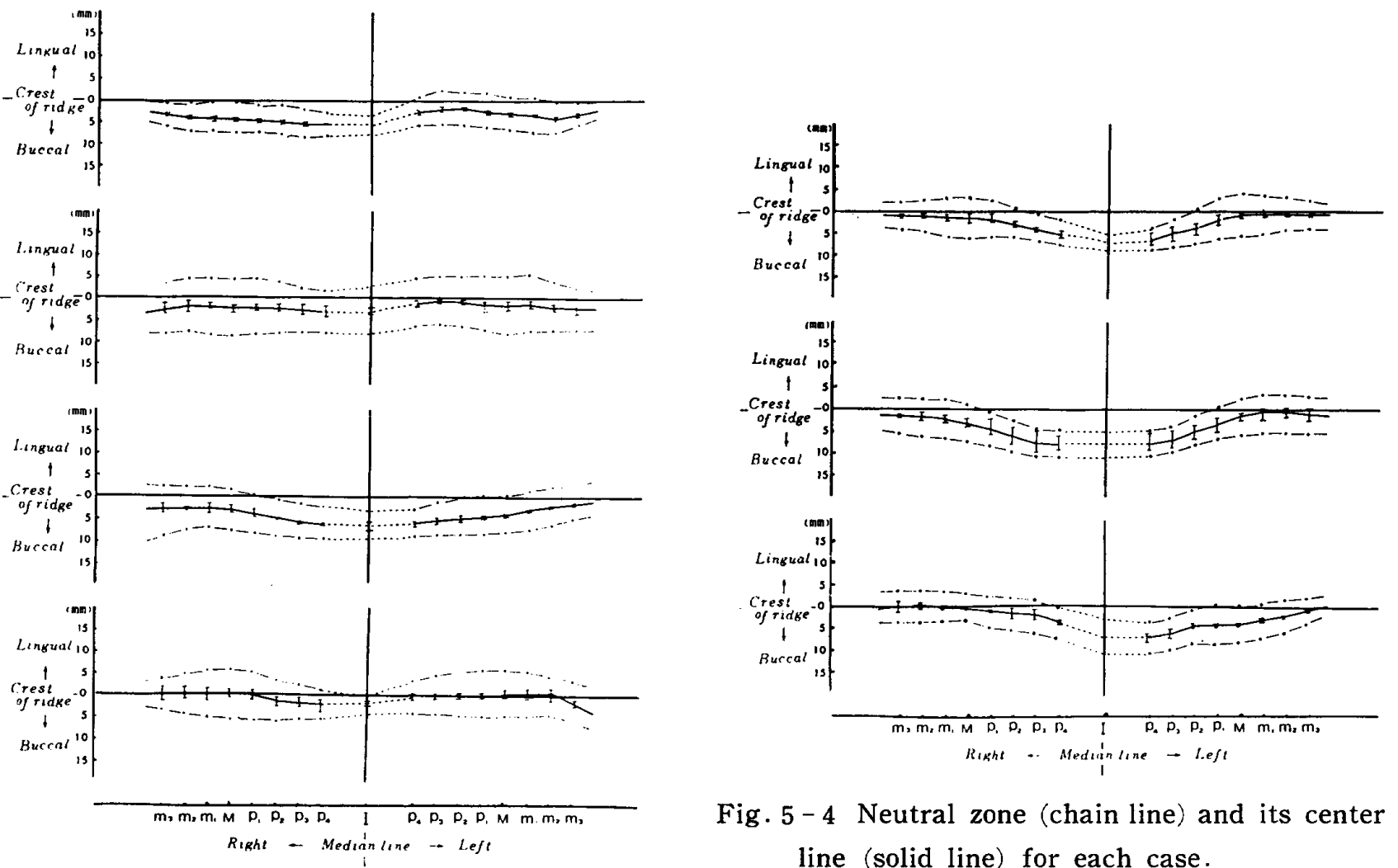

Fig. 5-4 Neutral zone (chain line) and its center line (solid line) for each case.

Fig. 5-2 Neutral zone (chain line) and its center line (solid line) for each case. 
5-4. Average distance of the mid-point from the crest was $4.66 \mathrm{~mm}$ on the reference point I. Extremcly large differences in the distance were found among patients, and in some cascs the mid-point was more than $10 \mathrm{~mm}$ apart from the crest to the labial side. In the molar region, the mid-point became closer to the alveolar crest and the diffcrences among the patients were not so marked as those in the incisal region. The average distance between the mid-point and the crest was $1.87 \mathrm{~mm}$. The deviation of the mid-point by repetition in each patient was also found to be larger in the incisal rcgion than in the molar.

The width of the neutral zone record, on the contrary, deviated more widely by repetition in the molar region than in the incisal (Table 4 ). The table showed a general tendency in the molar region that the width was slightly larger in the left side than in the right, and its deviation appeared to be also slightly larger in the left side than in the right.

Fig. 6 illustrates the plots of the mid-point $\bar{I}$ in the incisal region versus $\bar{M}$ in the molar of each neutral zone record. Positive correlation was recognized by regression analysis between them $(p<0.01)$. Similar correlation was obtained in the width between the reference points $I$ and $M(p<0.01)$ as shown in Fig. 7 . No relationship, however, could be detected between width and mid-point at the corresponding reference point

(Fig. 8 ).

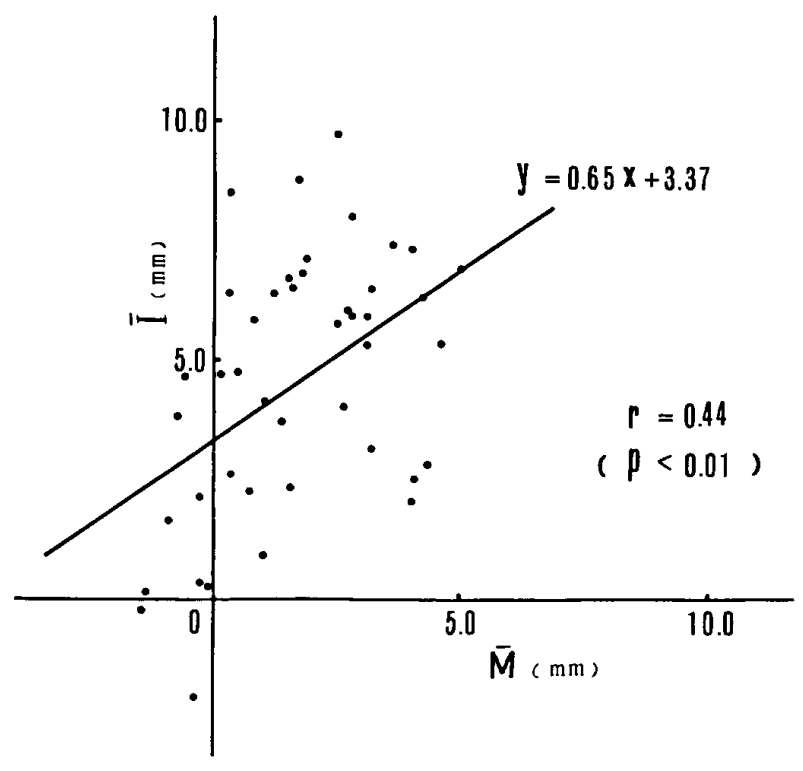

Fig. 6 Distance of the mid-point $\overline{\mathrm{I}}$ from the alveolar crest vs. distance of $\overline{\mathrm{M}}$.

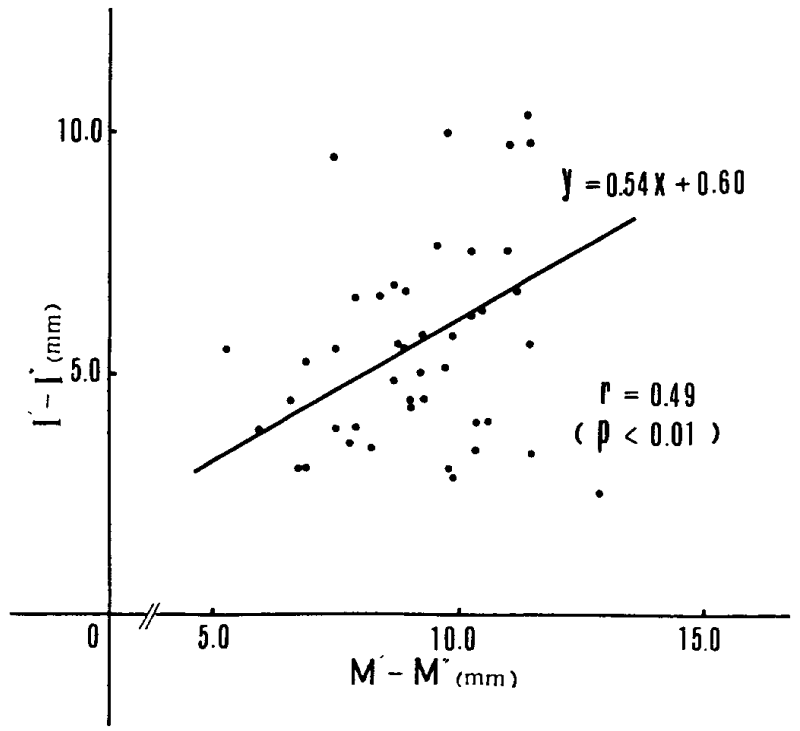

Fig. 7 Width at $I$ vs. width at $M$.

Fig. 9 shows the plots of the area $\mathrm{Sl}$ versus $\mathrm{Sb}$, and Fig.10 shows the plots of $\mathrm{Sl}$ and $\mathrm{Sb}$ versus $\mathrm{Sr}$. In these figures, $\mathrm{Sl}$ is the area surrounded by the straight line between the right and left retromolar pads and lingual margin of the neutral zone, $\mathrm{Sb}$ is the area surrounded by the same line and the labial, buccal margin, and $\mathrm{Sr}$ is the one similarly surrounded by the line and the alveolar crest. Sl was well correlated 


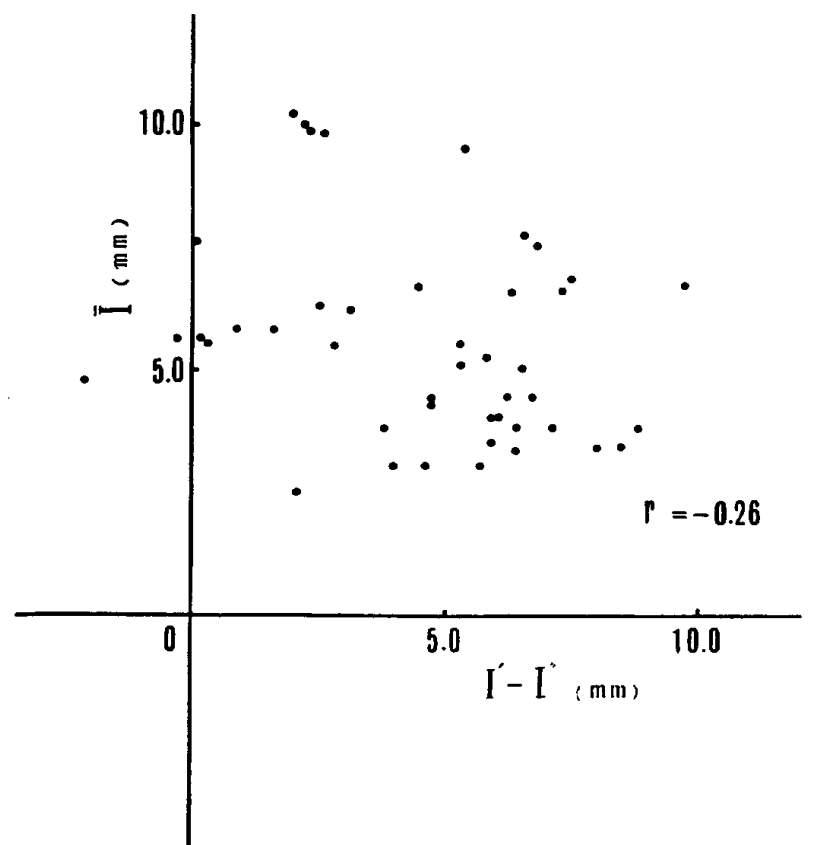

Fig. 8 Distance of the mid-point from the alveolar crest vs. width at the same reference point I.

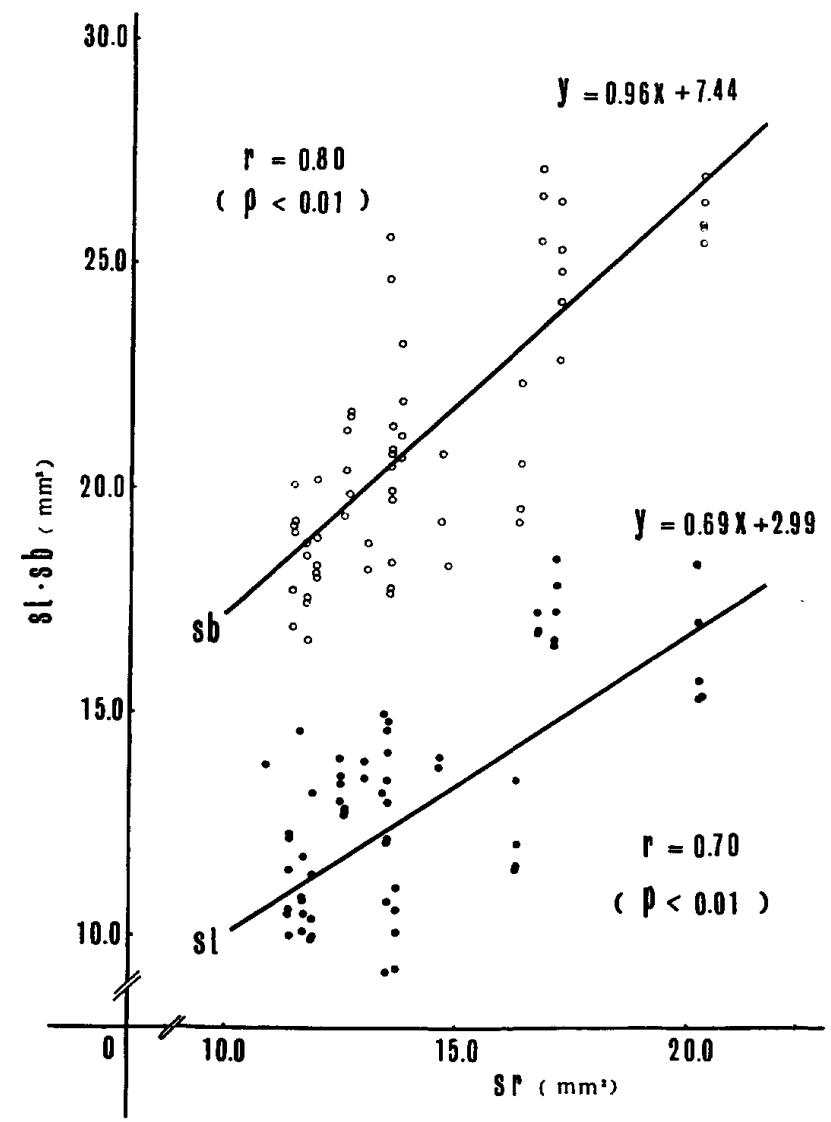

Fig.10 Areas $\mathrm{Sl}$ and $\mathrm{Sb}$ in Fig. 9 vs. area surrounded by the same. line and the alveolar crest, $\mathrm{Sr}$.

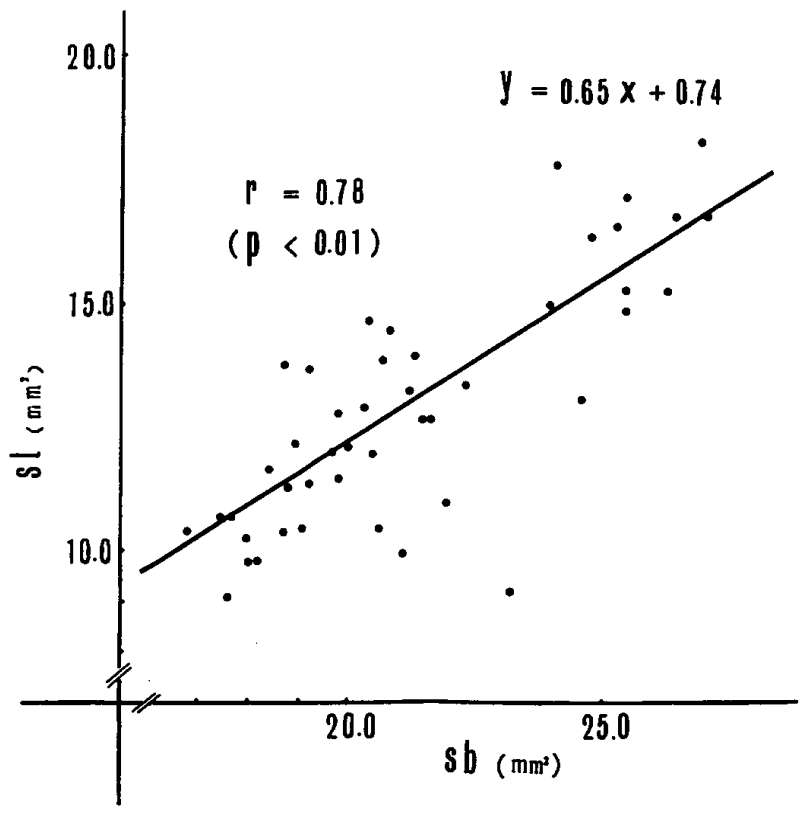

Fig. 9 Area surrounded by the straight line between the right and left retromolar pads and the lingual margin of the neutral zone, $\mathrm{Sl}$, vs. area surrounded by the same line and the labial, buccal margin, $\mathrm{Sb}$.

with $\mathrm{Sb}(\mathrm{p}<0.01)$ and both of them became larger as Sr became larger ( $p<$ 0.01). Correlation coefficient of $\mathrm{Sb}$ with Sr was somewhat higher than that of Sl.

The mid-point and the width of the neutral zone at the reference point I and $M$ were respectively plotted against the area $\mathrm{Sr}$ in Figs. 11 to 14 . In the case having larger $\mathrm{Sr}$, the mid-point of the obtained neutral zone record became closer to the alveolar crest while the width was independent of the area $\mathrm{Sr}$.

The center line of the neutral zone record lay on the labial and buccal side of the alveolar crest in the incisal and molar regions in most cases as seen in Figs. 5-1 to 5-4. A few cases showed the center line running across the crest from the buccal side into the 


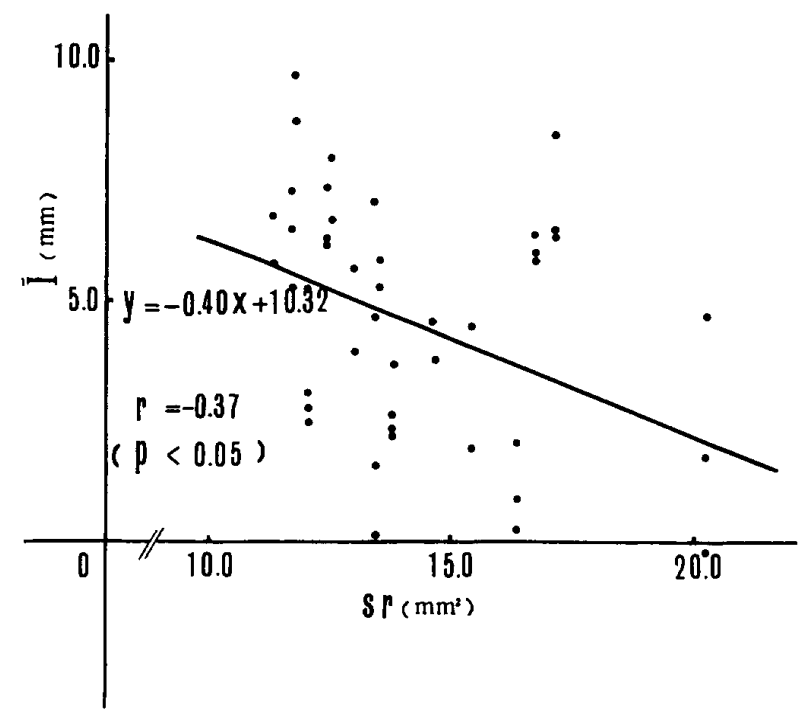

Fig.11 Mid point $\overline{\mathrm{I}}$ vs. area $\mathrm{Sr}$.

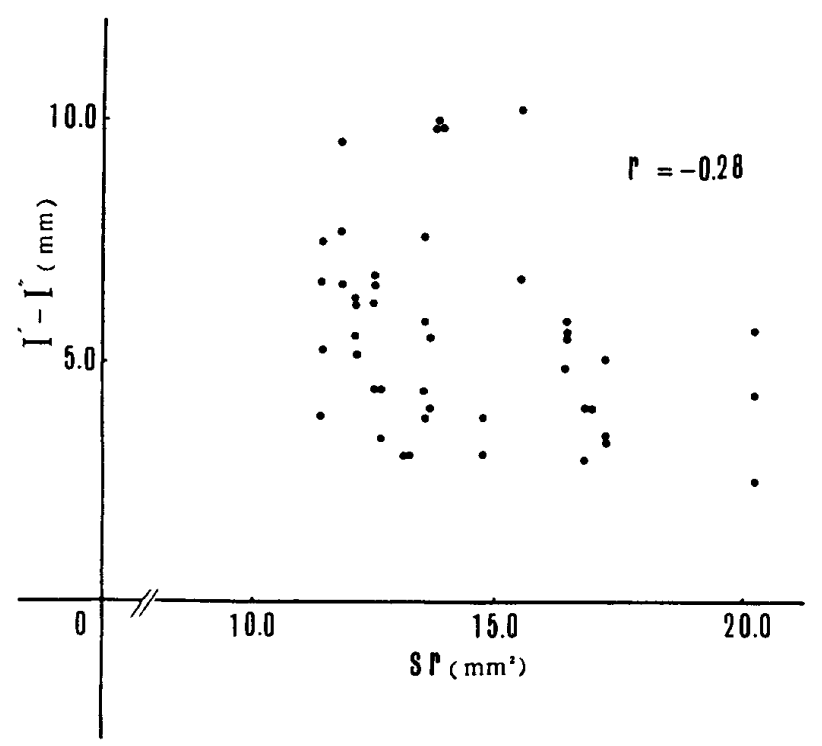

Fig.13 Width at I vs. area Sr.

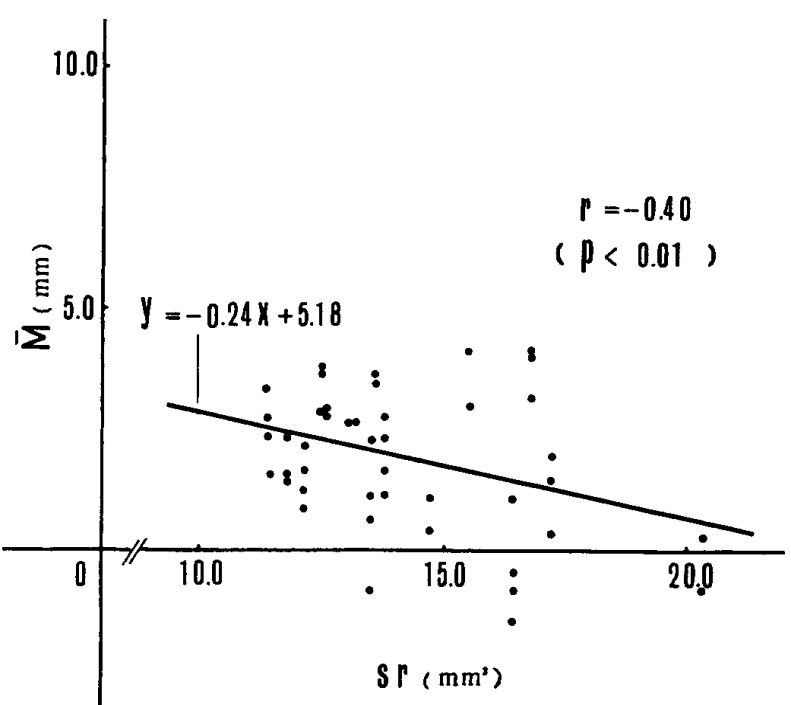

Fig.12 Mid-point $\overline{\mathrm{M}}$ vs. area $\mathrm{Sr}$.

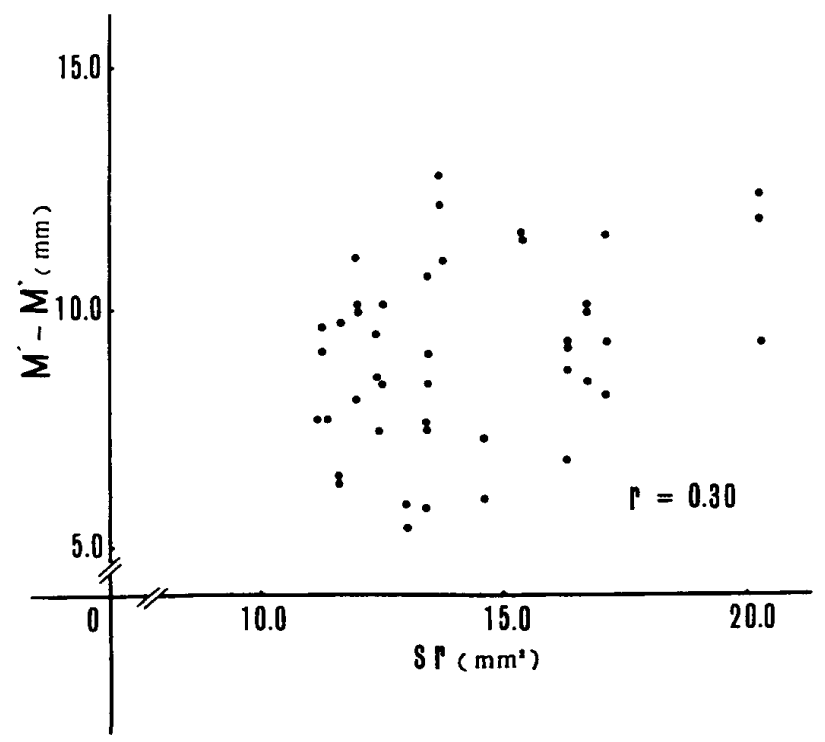

Fig.14 Width at $\mathrm{M}$ vs. area Sr.

lingual side at the molar region. The lingual margin of the neutral zone was generally on the lingual side of the alveolar crest in the region posterior to the point $M$, while in the incisal region it was found on the labial side in twelve cases out of fifteen. The intersecting point of the margin with the alveolar crest was distributed variously between the points $I$ and $M$. In the rest cases, the alveolar crest was fully covered with the neutral zone throughout the whole region.

Fig. 15 shows an example of the neutral zone records which were individually taken by two different practitioners for one patient. No statistically significant differences could be found in mid-point and width of the neutral zone between the two different practitioners.

Fig. 16 shows an example of illustrating the effects of the recording condition on 


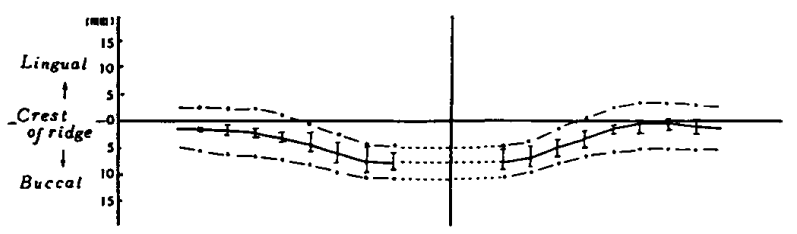

(a)
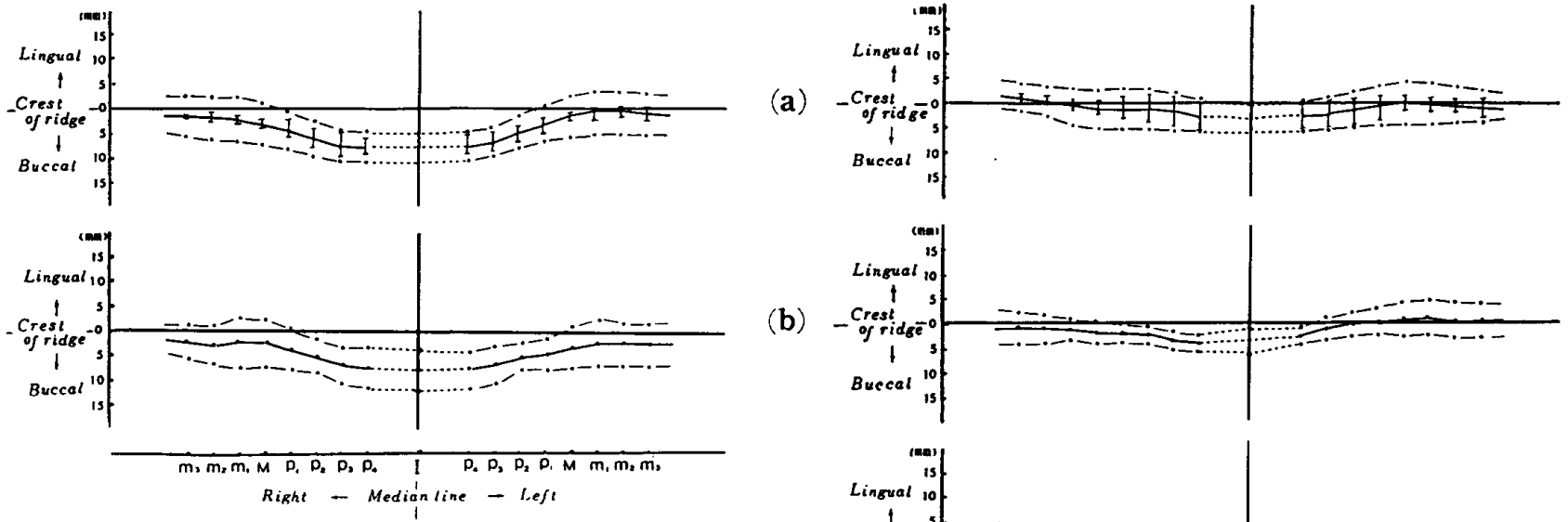

Fig.15 Example of the neutral zone records taken by two different practitioners for one patient.

the neutral zone. (a) is the neutral zone recorded under the normal condition as a control. When the recording was made under the condition of the elevated vertical dimension of occlusion by $5 \mathrm{~mm}$ at the anterior (b), the mid-point of the neutral zone was displaced to the labial and buccal side as compared with that of the control. Such a displacement was observed in almost all the cases examined. (c) was recorded utilizing

(d)

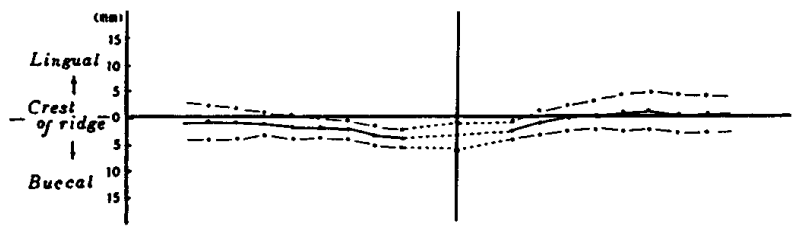

(c)
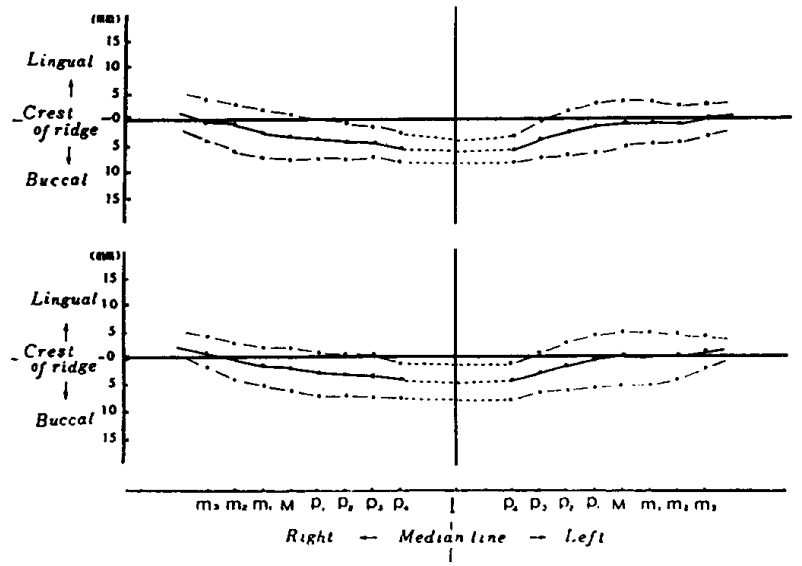

Fig.16 Example of the neutral zone records taken under altered conditions for one patient.
(a) normal condition
(b) elevating vertical dimension
(c) utilizing accustomed upper denture
(d) shifting the keels to the buccal side the accustomed denture in maxilla. The width of the neutral zone showed a tendency to become somewhat smaller than the control, while the mid-point was not changed markedly. When the keels were attached to the positions a little shifted to the buccal side (d), the resultant neutral zone was found to be slightly shifted to the labial and buccal side.

\section{Discussion}

The procedure of constructing the denture plate in neutral zone technique is carried out by strictly checking the stability of the plate in the mouth. Then it has been generally believed that the record of the neutral zone can be performed with excellent reliability and invariability by this technique, and that the record once made should not be modified in any way.

In this study, several numbers of the neutral zone records were made repeatedly under the same condition by an experienced practitioner of each patient. All of them were certified to be clinically satisfactory since the stability and other items had been severely checked on each trial by the practitioner and the patient. However, the 
values representing contour, size and site of the neutral zone varied with large deviation every time the neutral zone was recorded. Statistical analyses revealed that the reproducibility of the neutral zone recording in this technique was much poorer than ever expected.

The lack of the invariability of the mid-point was marked especially in the incisal region and that of the width in the molar in most cases. As the mid-point and the width were both calculated from the lingual margin and the labial or buccal margin, variations of the margins were directly reflected in the deviations of the mid-point and the width. In the molar region, the lingual margin and buccal margin deviated to a similar extent by repetition, resulting in large standard deviation of the width of the neutral zone without changing the mid-point largely. Such situations in the molar region may be reasonable if the neutral zone is to be formed as a result of balancing the opposite direction of the movements or forces in function between the tongue and the cheeks. Another tendency was found in the molar region that the right side showed somewhat smaller deviation than the left side. Most of the patients in this study had a habit of masticating on the right side, and it may be possibly considered that the tongue and the cheeks could easily maintain a stable relationship with each other on the right side because of the superior feed-back of the proprioceptive sensation on that side. In the incisal region, on the other hand, standard deviation of the lingual margin became larger than that of the labial margin. It seems to be due to the larger flexibility of the lingual apex in the functional movement. The difference in deviation between the lingual and the labial margins appeared to have changing effects mainly on the location of the mid-point but not strongly on the width.

The fact of the poor reproducibility of the neutral zone recording was seemingly discrepant with the clinical good performance in the neutral zone technique. It is assumed from such a discrepancy that the surrounding tissues may have some physiologic allowance for the denture plate in functional movement. On the basis of this assumption, any of the deviations can be considered to be within the allowance limit of the tissues and a case showing a wide deviation can be eventually identified with one having a wide allowance. It does not mean, however, that the neutral zone will cover the whole area from the maximum lingual margin to the maximum labial or buccal margin obtained by repetition in Table 1 and 2 . It should be noted that the neutral zone can exist somewhere within the deviation or the allowance limit having a cartain width shown in Table 4, since the neutral zone is formed by balancing the relative movements or forces of the tongue, lips and cheeks. The neutral zone recorded in the clinical practice would be nothing but one specific occupation area within the physiologic allowance. If it is tried to make the neutral zone again for the same patient, the new neutral zone may occupy the other area within the allowance where the denture plate will be sufficently stable. 
Sometimes in practice, the neutral zone as obtained may not satisfactorily be acceptable for teeth arranging by the sense of esthetics in anterior region. Only a slight alteration of the teeth positions may be possible at that time without serious loss of the adaptability to the surrounding tissues by taking advantages of the wide allowances, although the total balancing of the neutral zone should not be ignored. In those cases, however, it can not be confirmed whether the teeth are exactly within the allowance or not, until the stability is checked by constructing a wax denture with teeth arranged or a new neutral zone including the area of interest. Supposing that the stability of the denture is primarily important, it is of course essential to arrange the teeth within the obtained neutral zone as far as possible.

The center line of the neutral zone was located at $4.66 \mathrm{~mm}$ on average apart from the alveolar ridge crest to the labial side at the reference point $\mathrm{I}$, and $1.87 \mathrm{~mm}$ to the buccal side at $M$. It suggests that it will be difficult to expect the denture to be sufficiently stable in the mouth if the central grooves of the molar and premolar teeth are lined just above the alveolar crest.

Another factor of making the denture unstable is the unfit size of the teeth for the width of the neutral zone. Typical bucco-lingual width of the ready-made, first molar acrylic resin teeth of a commercial brand, for example, is 7.7 to $9.0 \mathrm{~mm}$, and that of the porcelain teeth is 7.4 to $8.3 \mathrm{~mm}$. Some of them are larger than the width of the neutral zone determined at the point $M$ in this study. The use of the teeth larger than the neutral zone width will result in poor adaptability of the denture to the surrounding tissues. Such an unfavorable relation will never be caused in the neutral zone technique, since the spaces for teeth arranging are definitely bound by the plaster matrices. If the denture is to be constructed without using this neutral zone technique, it is advisable to select relatively smaller size of the molar teeth and arrange them on the buccal side a little apart from the alveolar crest. Then, the teeth will likely get into the neutral zone area, and the resultant denture may occasionally have a chance to fit, though not completely, the functional movement of the surrounding tissues.

Although such a conventional method may be apparently easy and useful in some cases, the neutral zone technique will be indispensable in order to assure the best stability of the denture in the mouth. The lingual margin of the neutral zone was found to intersect the alveolar crest of the ridge in twelve cases out of fifteen as seen in Figs. 5 to 8 . It means that the alveolar crest does not run parallel to the neutral zone. As the intersecting point goes to the posterior, the alveolar crest becomes far apart from the center line of the neutral zone in the molar region. In those cases, therefore, the teeth positions will be liable to get out of the neutral zone area if the teeth are arranged according to the alveolar ridge line rule. In the neutral zone technique, on the contrary, the teeth will be arranged normally within the neutral zone contoured by the plaster matrices. Moreover, the teeth arranging procedure can be much easily performed, in which the teeth are only to be mechanically placed just 
along the matrices. It is one of the major advantages of this technique. The denture constructed in this way would be satisfactorily stable in the mouth because it can properly occupy the space where the forces of the tongue, lips and cheeks are neutralized in function.

Changing of the recording condition gave some effects on the neutral zone record. In comparison of the neutral zone records which had been separately taken by two different practitioners in several cases, no significant differences could be found between them. It indicates that the performance of the neutral zone may mainly depend on the practitioner's skill so far as he has experienced in this technique.

When the vertical dimension of occlusion is increased, the buccal mucosa will be stretched and the tongue room enlarged. It derives an anticipation that the inward forces by the cheeks will increase and the outward forces by the tongue will decrease against the recording material causing the displacement of the neutral zone toward the lingual side. The results, however, showed a contrary tendency in general. It seems that the increase of the vertical dimension of occlusion might not have simply reduced the tongue pressure due to the enlargement of the tongue room but yielded a different neutralizing situation such as the altered position and shape of the tongue in functioning in the enlarged room and the unussually frequent sucking because of the incresed saliva exudation.

If the accustomed upper denture is used in recording, it can be expected that the movement of the tongue will be smooth and natural. The smooth movement of the tongue might account for the slightly decreased width of the neutral zone under such a condition. This method appears to have an advantage to make the recording smoother and easier.

It was tried to shift the keels a little to the buccal side for one patient in this experiment to relieve the potential obstruction against the tongue movement. The resultant neutral zone was somewhat shifted to the labial and buccal side as compared with that obtained under the normal condition. In fact, some evidence that the keels might have obstructed the tongue movement are occasionally observed in practice on the neutral zone record. Positioning of the keels is considered to be one of the factors affecting the performance of the neutral zone.

Although reasonable changes were observed on the neutral zone record by altering the recording condition for some cases in this study, further investigations would be desired for more cases to certify these effects and improve the existing neutral zone technique.

\section{Summary}

Several numbers of the neutral zone records were made repeatedly under the same condition for each patient in this study. Although any of the neutral zone records was clinically judged to be satisfactory in function by the practitioner and the patient, it was found through the statistical analyses for labio, bucco-lingual mid-point and 
width of the neutral zone that the reproducibility of the neutral zone recording was much poorer than ever expected. Frequency of one record being significantly different in mid-point from other records obtained for the same patient was $55.4 \%$ in the incisal region and $66.4 \%$ in the molar. While in width, the frequency was $41.8 \%$ in the incisal and $49.1 \%$ in the molar.

The center line of the neutral zone record was located on the labial and buccal side of the alveolar crest in almost all the cases. Average distance of the mid-point from the crest was $4.66 \mathrm{~mm}$ in the incisal region and $1.87 \mathrm{~mm}$ in the molar. Deviation of the mid-point by repetition was found to be larger in the incisal than in the molar. Width of the neutral zone record, on the contrary, deviated more widely in the molar than in the incisal. Positive correlation was recognized by regression analyses between the positions of the mid-point in the incisal region and in the molar, and between the width of the neutral zone in the both regions. No relationships could be found between the values of the width and the mid-point at the corresponding reference point.

When the neutral zone records were made under some altered conditions, reasonable changes were observed on the records. No differences, however, could be detected between the different practitioners. It was found from this fact that the performance of the neutral zone record may mainly depend on the functional movements of the patient, but scarcely on the practitioner's skill so far as he has experienced in this technique.

\section{References}

1) Fish, E.W. : Analysis of stabilizing force in full denture construction. Brit. Dent. J. $52: 559$ $-570,1931$.

2) Fish, E.W. : Tongue space in full denture construction. Brit. Dent. J. 83:137-142, 1947.

3) Fish, E.W. : Principles of full denture prosthesis. Staples Press, London, 1964, 25-66, 87-102, $117-120$.

4) Lammie, G.A. : Aging changes and the complete lower denture. J. Prosthet. Dent. 6:450$464,1956$.

5 ) Rinaldi, P. and Sharry, J. : Tongue force and fatigue in adults. J. Prosthet. Dent. 13:857$865,1963$.

6 ) Raybin, N.H. : The polished surface of complete dentures. J. Prosthet. Dent. 13:236-239, 1963 .

7) Brill, N. and Tryde, G. : The dynamic nature of the lower denture space. J. Prosthet. Dent. $15: 401-418,1965$.

8 ) Pound, E. : Lost-fine arts in the fallacy of the ridge. J. Prosthet. Dent. 4:6-16, 1954.

9) Pound, E. : Recapturing esthetic tooth position in the edentulous patients. J.A.D.A. $55: 181$, 1957.

10) Pound, E. : Conditioning of denture patients. J.A.D.A. $64: 461-488,1962$.

11) Pound, E. : Preparatory dentures; A protective philosophy. J. Prosthet. Dent. 15:5-18, 1965.

12) Pound, E. : The mandibular movements of speech and their seven related values. J.Prosthet. 
Dent. $16: 835-841,1966$.

13) Pound, E. : Utilizing speech to simplify a personalized denture service. J. Prosthet. Dent. $24: 586-600,1970$.

14) Pound, E. and Murrell, G.A.: An introduction to denture simplification. J. Prosthet. Dent. $26: 570-580,1971$.

15) Pound, E. and Murrell, G.A. : An introduction to denture simplification, Phase II . J. Prosthet. Dent. $29: 598-607,1973$.

16) Tameyuki Yamamoto: Sohgishi kyushibu zinkohshi no hairetsu ni tsuite (I); Practice in Prosthodontics 5:131-136, 1972. (In Japanese)

17) Tameyuki Yamamoto: Sohgishi kyushibu zinkohshi no hairetsu ni tsuite (II) ; Practice in Prosthodontics 5:395-400, 1972. (In Japanese)

18) Beresin, V.E. and Schiesser, F.J.: The neutral zone in complete dentures; principles and technique. Mosby Company, 1973, 1-176.

19) Lott, F and Levin, B. : Flange technique; An anatomic and physiologic approach to increased retention, function, comfort, and appearance of dentures. J. Prosthet. Dent. $16: 394-413$, 1966.

20) Masaharu Tsubone and Shizuo Toyoda:Sohgishi Rinshoh Keitaigaku. Ishiyaku, Tokyo, 1978, 386-413. (In Japanese)

21) Atsushi Yamazaki : Bucco-lingual positional relation between neutral zone and alveolar ridge at the molar region of upper and lower edentulous jaws. J. Kyushu. Dent. Soc. 34:31-46, 1980 .

22) Shizuo Toyoda, Masao Morikawa, Shigeki Miyake and Toshihiro Shimizu : Transition of the apparatus for maintaining vertical dimension and characteristics of the resin post used for flange tecnique. J. Kyushu. Dent. Soc. $34: 512-517,1981$. 


\title{
仮想咬合平面上におけるニュートラルゾーンの再現性について
}

\author{
守川 雅雄 - 廖 梓琪 - 清水 稔弘 安元 和雄 - 豊田 静夫 \\ 九州歯科大学歯科補経学第 1 講座（主任：豊田静夫教授) \\ 小園 凱 夫 \\ 九州歯科大学歯科理工学講座（主任：林 一郎教授) \\ 佐藤 博 信 \\ 長崎大学歯学部歯科補綴学第 2 講匧（主任：藤井弘行教授）
}

ニュートラルゾーンテクニックは, ニュートラルゾー ン (以後, $\mathrm{N} \cdot \mathrm{Z}$ と記す) 内に適切に人工雷が排列さ れ,さらに義歯床辺縁および研磨面からの周囲組織の動 熊と緊密に適合することによって，はじめて効果的な義 歯の維持安定が確保されるという基本的理論に基づいた 術式であり，その成果は術者および患者の両者によって 大きく評価されている.

しかしながら， N・Zは歯牙の喪失および雬槽骨の吸 収によって生じた空隙であるために，それ自体が口腔内 で占める領域，形態は，一定であろうという既成概念が あり，その再現性つまり定形性等について行った根本的 な研究は見当らない.

この研究では, 同一条件下で, 同一術者が絽返えし記 録した場合の $N$ Z Z 部位および形態の動向と,さらに 条件を変えた場合について，仮想咬合平面上で検討し た。

当術式による $N$ ・ 記録の再現性は予期していたより はるかに小さく，同一思者における $N$ 技点の記録間
で統計学的に有意差の出る割合いは, 切歯部で55.4\%,

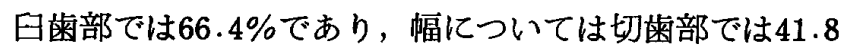
\%, 日畨部では49.1\%であった。

$\mathrm{N} ・ \mathrm{Z}$ 記録における煩舌的中央線はほとんどの症例で 雪槽頂より唇煩側に位置しており，その平均值は切歯部 で4.66mm, 曰歯部で1.87mm であった.また繰返えし による中点のバラッキは歯部より切䨑部で大きく，逆 に幅については切歯部より曰歯部が大きかった。

切歯部と日曾部の中点間ならびに幅間には正の相関関 係がみられたが，各それぞれ基準点における中点・幅間 には認められなかった。他方, 異った条件下でN・Z記 録を採得すると従来のものとの間にかなりの変化がるら れたが, 術者が代わっただけの条件の変化では著明な変 化はみられなかった。このてとはN・Zは主として患者 自身の機能運動によって記録されるものであり，ある程 度の経験さえあれば術者の巧拙にはよらないというとと を示唆していると考える. 